\title{
The effects of electrostatic forces on the distribution of drops in a channel flow: Two-dimensional oblate drops
}

\author{
Arturo Fernández ${ }^{\mathrm{a})}$ and Gretar Tryggvason ${ }^{\mathrm{b})}$ \\ Department of Mechanical Engineering, Worcester Polytechnic Institute, 100 Institute Road, Worcester, \\ Massachusetts 01609 \\ Judy Che and Steven L. Ceccio \\ Department of Mechanical Engineering and Applied Mechanics. University of Michigan, Ann Arbor, \\ Michigan 48109
}

(Received 10 March 2005; accepted 2 August 2005; published online 16 September 2005)

\begin{abstract}
Numerical simulations are used to examine the effect of an electrostatic field on an emulsion of drops in a channel. The leaky-dielectric theory of Taylor is used to find the electric field, the charge distribution on the drop surface, and the resulting forces. The Navier-Stokes equations are solved using a front-tracking/finite-volume technique. Depending on the ratios of conductivity and permittivity of the drop fluid and the suspending fluid the drops can become oblate or prolate. In addition to normal forces that deform the drops, tangential forces can induce a fluid motion either from the poles of the drops to their equator or from the equator to the poles. In this paper we focus on oblate drops, where both the dielectrophoretic and the electrohydrodynamic interactions of the drops work together to "fibrate" the emulsion by lining the drops up into columns parallel to the electric field. When the flow through the channel is slow, the fibers can extend from one wall to the other. As the flow rate is increased the fibers are broken up and drops accumulate at the channel walls. For high enough flow rate, when the drop interactions are dominated by the fluid shear, the drops remain in suspension. Only two-dimensional systems are examined here, but the method can be used for fully three-dimensional systems as well. (C) 2005 American Institute of Physics.
\end{abstract}

[DOI: $10.1063 / 1.2043147$ ]

\section{INTRODUCTION}

The behavior of multiphase flow is dominated by buoyancy for a wide range of operational conditions. In microgravity buoyancy is weak and surface tension often becomes the most significant force. Thus, electrostatic, thermocapillary, and acoustic forces can cause fluid flow and deformation of a fluid interface. To observe and study the motion of fluids in the presence of those weak forces, particularly for systems that are sufficiently large, require a high-quality microgravity environment that remains stable for a long time. Since such experiments are difficult and expensive, theoretical analysis is important. Analytical studies are, however, very limited in terms of geometrical complexities and numerical simulations are therefore the only realistic way of conducting theoretical studies. Recent progress in direct numerical simulations of multiphase flow, where all the details of the flow are fully resolved, has now made it possible to simulate the full unsteady motion of dispersed flows for a wide range of governing parameters. Such simulations have been used to examine the collective behavior of homogeneous bubbly flows and drops in channel flows. Simulations including secondary forces have, on the other hand, been limited to relatively small systems of one or two bubbles and drops. Here, the motion of an emulsion of drops in a channel

\footnotetext{
${ }^{a}$ Author to whom correspondence should be addressed. Present address: Department of Mechanical Engineering, The Catholic University of America, Washington, DC 20064. Electronic mail: fernanda@cua.edu

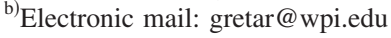

flow subject to electrostatic forces is examined, using direct numerical simulations, where the effects of inertia, viscosity, surface deformation, and electrohydrodynamic stresses are fully accounted for.

When a drop of one dielectric fluid suspended in a different dielectric fluid is exposed to an external, uniform electric field, the dielectric mismatch between the fluids induces a stress at the interface. In the case of perfect dielectrics and conductors, the electric surface force acts perpendicular to the surface and points from the fluid of higher dielectric constant to the lower. Changes in the shape of the interface and surface tension balance these induced electric forces. With just normal forces at the interface, an isolated drop can only deform into a prolate spheroidal shape. ${ }^{1-3}$ However, the experiments of Allan and Mason ${ }^{4}$ showed that an applied electric field deforms some drops into an oblate shape. The explanation for this effect was given by Taylor, ${ }^{5}$ who pointed out that the suspending fluids are not perfect insulators and that even a small finite conductivity allows charge to accumulate at the interface, resulting in a tangential electrical stress and motion of the fluid along the drop boundary. The process results in a flow recirculation inside and outside the drop; the direction and intensity depend on the electric properties of both fluids and the strength of the electric field. Taylor ${ }^{5}$ proposed a mathematical model to describe the motion, known as the leaky-dielectric model, and was able to predict qualitatively the deformation of the drops using a linear theory. Torza et al. ${ }^{6}$ reported the first quantitative experimental results on the deformation of single drops but the 
experiments showed considerable discrepancy between the measured deformations and those predicted by the linear theory of Taylor. Extending the theory to include higherorder terms ${ }^{7}$ did not improve the agreement with observations. In order to address the difference between previous experiments and theory, Vizika and Saville ${ }^{8}$ studied the deformation of both oblate and prolate single drops in steady and oscillatory electric fields. They measured directly the physical properties of the fluids and found a better agreement between the experimental and analytical results than did Torza et al. ${ }^{6}$ although a large discrepancy persisted in some cases. In order to explain the differences between the experimental and analytical results several explanations have been proposed. The most important source of error appears to be the difficulty in measuring accurately the electric properties of the fluids, especially the very low conductivities that these fluids usually have. This seems, in particular, to have been the case for the experiments done by Torza et al. ${ }^{6}$ Vizika and Saville $^{8}$ stated that other potential reasons for the discrepancies are inhomogeneity of the electric-field and changes of the electric field properties close to the surface of the drop. Although some uncertainty still exists, it seems fair to conclude that it is now widely believed that the leaky-dielectric model correctly accounts for experimental results, although direct comparisons have only been made for a relatively limited number of situations and more experiments are still necessary.

The dynamics of particles and drops in a channel, in the absence of an electric field, has been examined by several investigators. For one drop or particle, the behavior is reasonably well understood in the limit of zero Reynolds numbers. See Ref. 9 for a review. Computations of a single solid particle in a channel flow have been done by Feng et al. ${ }^{10}$ and Mortazavi and Tryggvason ${ }^{11}$ examined the motion of a deformable drop. Both authors examined finite Reynolds number flows and generally found good agreement with theoretical predictions for spherical particles. ${ }^{12-14}$ Both the drops and the particles did, in particular, generally settle down approximately half way between the channel centerline and its wall (the so-called Segre-Silverberg effect). As the Reynolds number was increased, Mortazavi and Tryggvason ${ }^{11}$ found that the droplets did not settle down, but exhibited periodic oscillations. Numerical studies of suspensions of particles started with Brady and Bossis, ${ }^{15}$ and Nott and Brady ${ }^{16}$ who used Stokesian dynamics to follow the evolution. Zhou and Pozrikidis ${ }^{17}$ used a boundary integral method to follow the motion of many two-dimensional deformable drops. Boundary integral simulations of threedimensional drops have been done by Loewenberg and Hinch. ${ }^{18}$ So far the focus has been nearly exclusively on Stokes flow where it is generally found that the parabolic laminar profile for flow without drops is blunted as the drops move towards the center of the channel. For a recent discussion see Ref. 19. For a review of inertial effects in suspensions in general, see Ref. 20.

While computational studies of electrohydrodynamics of drops have been limited to one or two drops, a few authors have simulated numerically the behavior of a suspension of solid particles in a nonconducting fluid in the presence of an electric field, with an emphasis on the bulk properties. The electrorheology of solid particle suspensions was first studied by Winslow ${ }^{21}$ and has been reviewed by, for example, Block and Kelly, ${ }^{22}$ Hulsey et al., ${ }^{23}$ and Hulsey ${ }^{24}$ who discuss the experimental and analytical results. Such suspensions generally show fibration (or fibrillation) where a polarization of the particles leads them to line up across the channel. The formation of fibers generally increases the bulk viscosity of the suspension greatly. Klingerberg et al. ${ }^{25,26}$ used a molecular-dynamics-like methodology to examine the behavior of electrorheological suspensions without and with an applied shear. In spite of the simplicity of the model, which neglected local changes in the electric field due to the presence of the particles, the results showed qualitative and, to some degree, quantitative agreement with experimental results. An improved method, based on Stokesian dynamics, was used by Bonnecaze and Brady ${ }^{27}$ to examine the effect of shear, but in the absence of walls.

Arp et $a .^{28}$ provided a comprehensive review of early progress in the understanding of the response of suspensions of both drops and solid particles to electric fields, emphasizing how the microstructure influenced the bulk properties. Recent experimental work by Pan and McKinley, ${ }^{29}$ Kimura et al. ${ }^{30}$ and $\mathrm{Ha}$ and $\mathrm{Yang}^{31}$ has helped clarify many aspects of the behavior of emulsions. These authors have examined the electrorheological response of emulsions consisting of several different fluids and observed fiber-like chains of drops parallel to the electric field. Pan and McKinley ${ }^{29}$ were the first authors to measure the shear stresses in an emulsion for different electric-field strengths and void fractions. They found that the response of their emulsion-consisting of paraffin oil and silicone oil — was strongly dependent on which fluid was the dispersed phase, but the effective viscosity always increased with an increase of the electric field, an effect usually referred to as positive electrorheological behavior. Kimura et al. ${ }^{30}$ presented the results for an emulsion glycoldimethylsiloxane. They experimented with different viscosity ratios between the fluids, and found that for drops with a lower dielectric constant than the suspending fluid, a negative electrorheological behavior is possible when the viscosity of the continuous phase is much larger than the viscosity of the drops. They attributed this effect to the fact that when the drops are suspended the viscosity depends mainly on the continuous phase, but when the fibers appear the bulk viscosity is dominated by the force needed to break up the fibers, and this force depends on the viscosity of the second phase. Ha and Yang ${ }^{31}$ prepared an oil-in-oil emulsion where not only the viscosity, but also the conductivity ratios, could be modified. They found a negative electrorheological behavior, although this effect was seen for viscosity ratios higher than unity, and the intensity of the negative electrorheological behavior was much stronger than what was observed by Kimura et al. ${ }^{30}$ Their explanation for this phenomenon relies on the electrorotation of the drops due to electrokinetic effects. In a parallel effort, the group directed by Orihara has studied the behavior of a polymer blend in a shear flow, see Refs. 32-35. They studied how the shape and elongation of the second phase depend on the relative strength between shear and electric field. 
The first numerical simulation of the effect of electric fields on a single drop was done by Sherwood ${ }^{36}$ who used a boundary integral method to examine the deformation and breakup of a single drop in the Stokes flow limit. Tsukada et $a l^{37}$ studied the circulation induced inside and outside a single suspended drop using a Galerkin finite element method. They found good agreement with Taylor's analytical results and their own experiments. In a later paper, Tsukada et $a l^{38}$ studied the combined effect of gravity and electrohydrodynamical forces. They found that the terminal velocity is only very slightly modified by the presence of the electric field but that the electric forces result in the appearance of a wake behind the drop, even for small Reynolds numbers $(\operatorname{Re}<1)$. Feng and $\mathrm{Scott}^{39}$ simulated the deformation of a single drop in both the Stokes limit and at finite Reynolds number using a Galerkin finite element methods similar to that of Tsukada et al. ${ }^{37,38}$ The main conclusion is that for small deformations, the numerical results agree with the asymptotic solutions of Taylor. ${ }^{5}$ However, a large discrepancy appears when the deformation of the drop is large enough. The two-drop problem has been investigated by Sozou $^{40}$ in the limit of negligible drop deformations with no relative motion between drops. A very comprehensive study of the axisymmetric interaction of two drops, in the limit of zero Reynolds number, was done by Baygents et al., ${ }^{41}$ using a boundary integral technique. They showed that the electrohydrodynamically induced flow could significantly modify the dielectrophoric attraction, either acting with it or against.

The main issue that is addressed by the present work is the response of an emulsion of drops in a channel when an electric potential difference is applied between the channel walls. Before presenting the results for the channel flow, we show the interaction between two drops and relate them to the results obtained by Baygents et al. ${ }^{41}$ This interaction depends on the viscous fluid motion induced by the electric field and the dielectrophoretic effect resulting from the drops acting like dipoles.

The drop distribution depends strongly on the dielectric properties of both fluids. In this paper, we present only results for two-dimensional flows. The advantage of the twodimensional simulations is that it allows us to follow the long-time drop evolution, without employing very timeconsuming three-dimensional simulations. The influence of several parameters such as the relation between the electrostatic forces and the shear, the electric-field strength, and the volume fraction is also quantified.

\section{FORMULATION}

The physics governing electrohydrodynamics of multiphase flows has been reviewed by several authors including Melcher and Taylor ${ }^{42}$ and Saville ${ }^{43}$ who discussed the leakydielectric model proposed by Taylor ${ }^{5}$ for fluids that have a small but finite conductivity. Scaling analysis shows that it is appropriate to assume a quasistatic electrostatic field for a fairly general class of situations and find the electric potential $\phi$ by solving the steady-state conservation of charge equation $\nabla \cdot \sigma \nabla \phi=0$.

Here $\sigma$ is the electric conductivity and the electric field $\mathbf{E}$ is obtained from the electric potential $\phi$ by

$$
\mathbf{E}=-\nabla \phi .
$$

Once the electric field is found, the free charge is obtained by Gauss' law:

$$
q=\nabla \cdot \varepsilon \mathbf{E} .
$$

Here $\varepsilon$ is the electric permittivity of the fluid. These equations assume that the materials in both phases have a finite dielectric coefficient and conductivity.

Following Melcher and Taylor ${ }^{42}$ the forces acting on the boundary between both fluids can be obtained from the jump in the electric stresses. The final result, for the force per unit volume, is

$$
\mathbf{F}_{\text {elec }}=q \mathbf{E}-\frac{1}{2}(\mathbf{E} \cdot \mathbf{E}) \nabla \varepsilon+\nabla\left(\frac{1}{2} \mathbf{E} \cdot \mathbf{E} \frac{\partial \varepsilon}{\partial \rho} \rho\right) .
$$

If $\sigma$ and $\varepsilon$ are constant in each fluid the force only acts on the phase boundaries. The first term in Eq. (4) represents the contribution of free charges to the force and the second term is due to the polarization force density. The last term results from changes in the material density, usually called electrorestriction force density. This term is neglected in the present work. Perfect dielectric and leaky-dielectric fluids experience charge polarization when immersed in a uniform, external electric field. However, leaky-dielectric fluids show an accumulation of charges at the surface that modifies the electric field. This accumulation of charges, coupled with the tangential electric stresses, generates tangential forces at the interface that eventually lead to the viscous fluid motion seen for these fluids.

The fluid flow is governed by the Navier-Stokes equations,

$$
\begin{aligned}
\frac{\partial \rho \mathbf{u}}{\partial t}+\nabla \cdot \rho \mathbf{u u}= & -\nabla p+\left(\rho-\rho_{o}\right) \mathbf{g}+\nabla \cdot \mu\left(\nabla \mathbf{u}+\nabla \mathbf{u}^{T}\right) \\
& +\gamma \int k^{\prime} \mathbf{n}^{\prime} \delta^{\beta}\left(\mathbf{x}-\mathbf{x}^{\prime}\right) d A+\mathbf{F}_{\text {elec }} .
\end{aligned}
$$

Here, $\mathbf{u}$ is the velocity, $P$ is the pressure, and $\rho, \mu$, and $\gamma$ are the density, viscosity, and surface tension. The buoyancy term has been neglected and the only new term is the electric force $\mathbf{F}_{\text {elec }}$ that is calculated by Eq. (4). The momentum equation is supplemented by an equation of mass conservation. If both fluids are incompressible it is

$\nabla \cdot \mathbf{u}=0$.

The numerical method used in this study is the finitevolume/front-tracking method developed by Unverdi and Tryggvason. ${ }^{44}$ A review of the method can be found in Tryggvason et al., ${ }^{45}$ along with several validations and examples. The momentum equation is discretized on a regular staggered grid using second-order, centered finite differences for the spatial derivatives and a second-order projection time integration scheme. The continuity equation, combined with the momentum equation, results in a pressure equation that is 
solved by a multigrid scheme. The charge conservation equation (1) is discretized using standard-centered differences and solved by the same multigrid scheme used for the pressure equation. The electric potential is obtained at the center of the pressure cells and the electrostatic forces [Eq. (4)] can be found directly at the edges the cells where the velocity is stored. The electrostatic forces, Eq. (4), are added directly to the predictor step in the projection method used to integrate the momentum equations in time.

The most important aspect of numerical methods for solution of multiphase problems is the correct tracking of the interface. In the front-tracking method the boundary between the drops and the continuous fluid is represented by connected marker points (the "front"), which are advected by a velocity interpolated from the fixed grid. Here we used the interpolation function presented by Peskin ${ }^{46}$ both to interpolate the velocities as well as to distribute surface forces onto the fixed grid. A few simulations were also done using area weighting and no major differences were found. If the front stretches or is compressed too much, new elements are added or old ones are removed, respectively, in order to maintain an accurate representation of the interface.

For a Poiseuille flow, if no electrohydrodynamical effect is included, the drop-laden flow can be completely characterized by specifying the Reynolds and the capillary numbers

$$
\mathrm{Re}=\frac{\rho_{o} U H}{\mu_{o}}, \quad \mathrm{Ca}=\frac{\mu U}{\gamma},
$$

in addition to the density and viscosity ratios, $r=\rho_{i} / \rho_{o}, \lambda$ $=\mu_{o} / \mu_{i}$, respectively, and the void fraction $\alpha$. Here, the Reynolds and the capillary numbers are defined using the velocity of the centerline, $U$, that would result from the applied pressure gradient if there were no drops. $H$ is the height of the channel and $a$ is the drop radius. As reference density and viscosity the properties of the suspending fluid have been used. When electrostatic effects are included three new dimensionless parameters are introduced. The first two are the ratios of the electric permittivities and conductivities $1 / S$ and $R$, defined as $1 / S=\varepsilon_{i} / \varepsilon_{o}$ and $R=\sigma_{i} / \sigma_{o}$, respectively. The subscript $i$ refers to the drop phase, while $o$ refers to the suspending fluid. The third one describes the strength of the electric field. There are several possible choices for this parameter, including the inverse of the nondimensional electrostatic force that relates the electrostatic force to the advection term,

$$
\frac{1}{E^{*}}=\frac{\text { Advection term }}{\text { Electric force }}=\frac{\rho U^{2}}{S^{-1} \varepsilon_{o} E_{\infty}^{2}},
$$

and the electric capillary number,

$$
C_{e}=\frac{\text { Electric forces }}{\text { Surface tension }}=\frac{S^{-1} \varepsilon_{o} E_{\infty}^{2} a}{\gamma},
$$

which relates the electrostatic forces to the surface tension and helps to predict the deformation of the drops. In these equations, $E_{\infty}$ is the uniform electric field that results from the applied electric potential difference between the walls in the case of a uniform fluid. For the problem that we are examining, we want to contrast the behavior with and with- out flows, so we need to be able to describe the fluid without the electric field without reference to the flow velocity. The Ohnsorge number,

$$
\mathrm{Oh}=\frac{\mathrm{Re}}{\sqrt{\mathrm{We}}}=\frac{\mu_{o}}{\sqrt{\gamma \rho_{o} a}},
$$

allows us to do that. The effect of the electric field on the drops is then described by the electric capillary number (9) and the inverse of the nondimensional electrostatic force (8) to quantify the strength of the flow. Thus, our set of nondimensional numbers is $\mathrm{Oh}, C_{e}$ and $1 / E^{*}$. For the study of the effect of the flow on the motion of the drops, $E^{*}$ is the only nondimensional number that is changed.

The main assumption in the leaky-dielectric model is that the electric relaxation is much faster than the convection of charges by the flow. To measure the relative importance of those effects the electric Reynolds number $\left(\operatorname{Re}_{E}\right)$ is defined as the ratio of the time scale of the charge relaxation and the time scale of the charge convection by the flow. The resulting expression is

$$
\operatorname{Re}_{E}=\frac{9\left(\varepsilon_{o}+\varepsilon_{i}\right)}{10\left(\sigma_{o}+\sigma_{i}\right)}\left(\frac{\left|R \cdot S^{-1}-1\right|}{(2+R)^{2}(1+\lambda)}\right) \frac{\varepsilon_{i} E_{\infty}^{2}}{\mu_{i}} .
$$

In the simulations presented here, $\mathrm{Re}_{E}$ is low enough so that charge relaxation can be assumed to be unimportant and the leaky-dielectric model should be valid. For the worst case presented in this paper $\operatorname{Re}_{E}$ is about 0.0375 . Krause and Chandrateya ${ }^{47}$ presented an experimental study of the electrorotation of drops and defined an electric-field threshold for the rotation of the drops caused by electrostatic convection,

$$
E_{\text {threshold }}^{2}=\frac{2 \lambda(1+2 R)^{2}}{3 \varepsilon_{i} S^{-1} R\left(R \cdot S^{-1}-1\right)} .
$$

In all our simulations this criteria is satisfied. However, our highest electric-field strength corresponds to half the threshold value, which means that in the most unfavorable case we are very close to the limit. An increase in the electric-field strength or in the conductivity of the fluids would cause electroconvection effects to be relevant, and a conservation equation for the electric charges would have to be added to the system.

To render time nondimensional, it is also necessary to define an electric time scale. Because the viscous fluid motion depends on the viscosity of the flow and the intensity of the electric field, we define the following electric time scale:

$$
\tau_{\mathrm{el}}=\frac{\mu}{\varepsilon_{o}\left(\varepsilon_{i} / \varepsilon_{o}\right) E_{\infty}^{2}} .
$$

Baygents $e t a l .{ }^{41}$ used the leaky-dielectric model to study the interaction between two leaky-dielectric drops in the limit of $\mathrm{Re} \rightarrow 0$. They point out that the interaction is the result of two different effects acting on both drops. There is an attractive dielectrophoretic force parallel to the electric field due to the fact that the drops see each other as dipoles in the far field. This force is proportional to $O(a / r),{ }^{4}$ where $r$ is the distance between the centers of the drops. The other force is either attractive or repulsive and is due to the viscous circulatory flow induced by the electric stresses. In a similar 
way as the deformation of a single drop, the interaction between two drops is governed by the competition between viscous fluid motion and dielectrophoretic effect. Oblate drops present a more uniform behavior because the viscous fluid motion always goes from the poles to the equator and leads to an attractive force in the direction parallel to the electric field, thus strengthening the dielectrophoretic attraction. However, the viscous fluid motion for prolate drops can go from the poles to the equator or from the equator to the poles. In the latter case the repulsive viscous and the attractive force oppose each other, resulting in a complex and rich behavior of prolate drops.

Leaky-dielectric drops interact with walls in qualitatively the same way as they do with each other. The viscous circulation induced by the electric stresses attracts or repels the approaching drop. Once a drop is attached to a wall, it is very difficult to break the bond due to the low velocity close to the walls.

Taylor ${ }^{5}$ obtained an analytical solution for the viscous fluid motion in the limit of zero Reynolds number. He found that the tangential component of the surface velocity, expressed in spherical coordinates, is

$$
u_{\theta}=-\frac{9\left(R-S^{-1}\right) \varepsilon_{o} E_{\infty}^{2} a}{5(1+\lambda)(R+2)^{2}} \cos \theta \sin \theta .
$$

Thus, the direction of the flow depends on the sign of $R-S^{-1}$. When it is positive the flow goes from the equator to the poles, while when it is negative the flow goes from the poles to the equator.

The deformation of drops is composed of viscous fluid motion and deformation caused by the dielectrophoretic effect. Taylor ${ }^{5}$ also obtained a discrimination function for a spherical drop,

$$
\Phi=S^{-1}\left(R^{2}+1\right)-2+\frac{3}{5}(S R-1) \frac{(2 \lambda+3)}{\lambda+1},
$$

and determined that if $\Phi$ is positive the drops are prolate, while $\Phi$ is negative for oblate drops. Rhodes et al. ${ }^{48}$ found a similar discrimination function for two-dimensional drops and $\lambda=1$ :

$$
\Phi_{2 \mathrm{~d}}=(R)^{2}+(R)+1-3 \cdot S^{-1} .
$$

Figure 1 shows the analytical function, $\Phi_{2 \mathrm{~d}}=0$, for zero deformation of the drops. The abscissa is the ratio between the dielectric permittivities $\left(S^{-1}\right)$, and the ordinate is the conductivity ratio $(R)$. The bold line represents the discrimination function, below it the drops become oblate and above it they become prolate. The viscous fluid motion goes from the poles to the equator below the dashed line, and the flow reverts from the equator to the poles above the dashed line.

\section{RESULTS}

\section{A. The deformation of a single drop and the interaction between two drops}

Before we address the interactions of two drops, we discuss briefly the effect of the electric field on one drop. A uniform electric field does not move a single isolated drop,

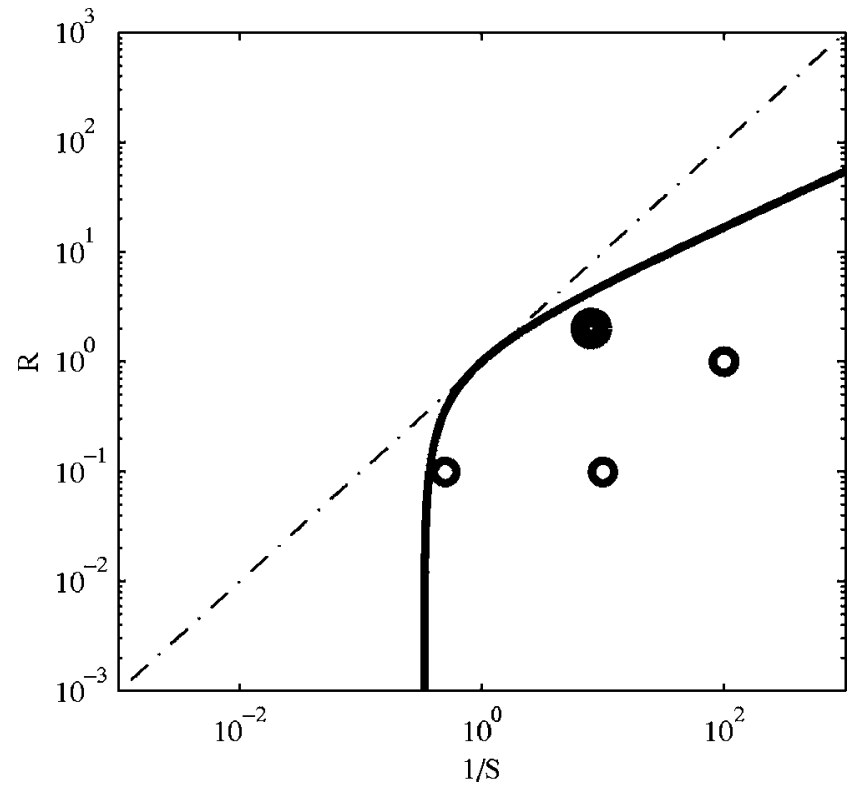

FIG. 1. Map of expected drop response based on the leaky-dielectric model. The bold line represents the discrimination function for a two-dimensional flow. A closed circle marks the case studied in this work, and the open circles mark other simulated cases.

but the drop deforms and the tangential force on the drop surface results in fluid motion both inside and outside the drop. When two drops are placed next to each other, the induced flow can modify the dielotrophoretic interaction in a significant way. Figure 2(a) shows the steady-state shape and streamlines for one two-dimensional drop and Fig. 2(b) shows the electric potential. Here the conductivity and permittivity ratios are $R=2$ and $S^{-1}=8$, respectively. A closed circle in the $S-R$ plane in Fig. 1 marks the location of this case. The drop is located in a channel that is periodic in the horizontal direction and has solid top and bottom boundaries. An electric potential difference is applied between the top and the bottom walls. The electric-field strength is quantified using the electric capillary number because no flow is imposed and here $C_{e}=1.32$. We note that this capillary number is larger than for most of the rest of the simulations in the paper to allow for relatively large deformation. The square computational domain, whose sides are two times the diameter of the drop, is resolved by a $64^{2}$ grid. The electric stresses induce a flow at the surface of the drop from the poles to equator and the drop becomes oblate. The deformation parameter is defined by

$$
\operatorname{Def}=\frac{L-D}{L+D},
$$

where $L$ is the height of the drop (parallel to the electric field), and $D$ is the diameter (perpendicular to the electric field). Def is plotted in Fig. 2(c) versus time for the drop in Fig. 2(a) as well as drops resolved on a $32^{2}$ grid, a $128^{2}$ grid, and a $256^{2}$ grid. While the deformation of the drop computed on the coarsest grid is significantly smaller than for the finer grids, the difference between the medium grid $\left(64^{2}\right)$ and the two finer grids $\left(128^{2}\right.$ and $\left.256^{2}\right)$ is relatively small, suggesting that the $64^{2}$ grid produces an essentially fully converged so- 

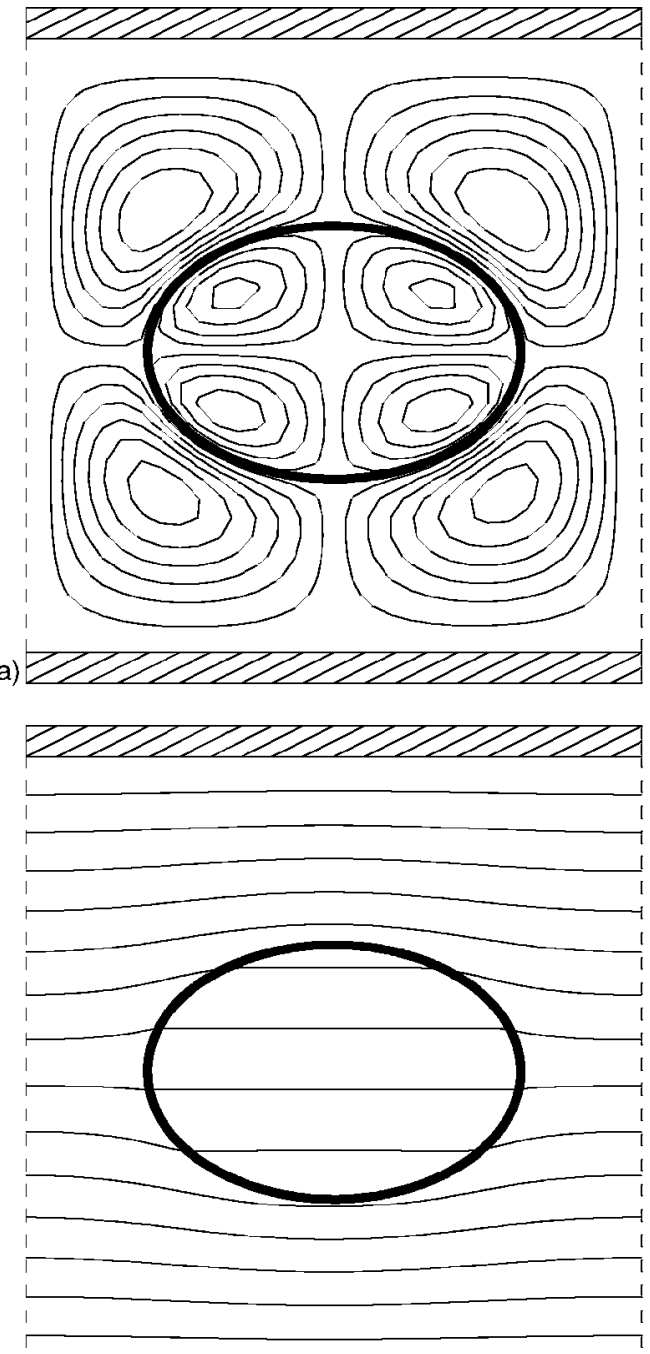

(b)
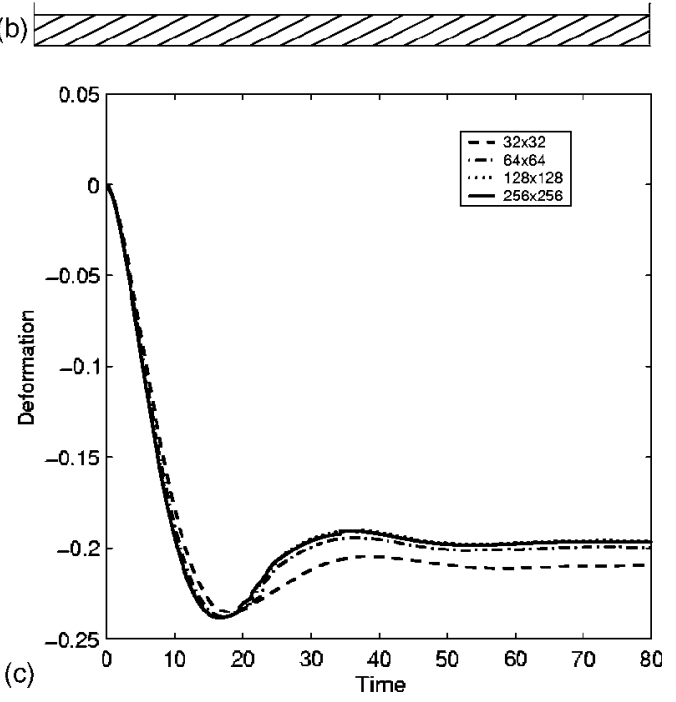

FIG. 2. (a) Drop deformation and streamlines for a single oblate drop immersed in an electric field. $S^{-1}=8.0, R=2.0$, and $C_{e}=1.32$. (b) The electric field for the drop shown in (a). (c) Resolution test for a single drop. Resolution of the grid: $32 \times 32$ (number of points per undeformed diameter $n_{d}$ $=16), 64 \times 64\left(n_{d}=32\right), 128 \times 128\left(n_{d}=64\right)$, and $256 \times 256\left(n_{d}=128\right)$.

lution. On this grid, the drop is resolved by 32 points across the diameter of the undeformed drop. We have simulated the deformation of several other oblate drops for different $S$ and
$R$ and find similar results in all cases. We have also simulated several drops close to where the discrimination function [Eq. (6)] is 0 and find that the boundary between oblate and prolate drops obtained computationally agrees well with analytical predictions. Other validation tests were done by $\mathrm{Che}^{49}$ who compared results for axisymmetric drops with the predictions of Feng and Scott. ${ }^{39}$ The general conclusion from her simulations, as well as from our tests, is that $20-30$ grid points per diameter yield accurate results over a wide range of parameters.

To examine the interaction between drops we have done several simulations where two drops are initially put close to each other. Figure 3 shows three frames from a simulation of the motion of two drops. The properties of the drops are similar to the case shown in Fig. 2, but the radius is smaller and $C_{e}=0.8$. The domain is, however, larger (the sides are 3.33 times the diameter of the original drop) and resolved by a $128^{2}$ grid. The drops are initially located near the center of the domain, but slightly closer to the bottom wall. The drop centers are about two-drop diameters apart and on a line at a $45^{\circ}$ angle with respect to the electric field. The first frame in Fig. 3 shows the drops near time zero where the electric field has set the fluid in motion but the drops have neither moved nor deformed to any significant degree. In the second frame, the induced circulation forces the drops to line up parallel to the electric field, while the distance between them decreases slightly. The drop closer to the bottom wall has also moved to the wall as the fluid motion induced at the drop surface drains the region between the drop and the wall. Once the drops have lined up, the fluid is drawn out from the region between them and they form a short fiber attached to the wall (frame 4). When the drops are close, they also attract each other because of the dielectrophoretic attraction.

To examine in more detail the interaction of two drops, we have simulated the motion of several drops for different initial orientations and the same electrical parameters. In Fig. 4(a) we plot, using a solid line, the vertical separation of the drops versus the horizontal separation for five different initial conditions. In three cases, where the initial separation is not too large, the drops first line up, sometimes first increasing their separation, and then move together to the wall along a line parallel to the electric-field lines. In the fourth case the drops are lined up in the direction of the electric field, but their initial separation is sufficiently large, so the drop-todrop interaction is very weak. The drops therefore move to the opposite walls. In the last case, the drops are lined up in a direction perpendicular to the electric field and the drops are repelled. We have repeated one of these simulations using coarser and finer grids. The results show that except for very low resolution the curves are essentially the same. The conclusion is similar to what was found for the tests using one drop: when the undeformed drop is represented by 20-30 grid points, a finer grid does not modify the results. When the resolution is lower, around 15 points per diameter, the final result does not change, but the film between the drops drains slightly faster.

In addition to the simulations in Fig. 4(a), which are for the same parameters as used in Fig. 3, we have done a number of simulations for other values of $S$ and $R$ that lead to an 


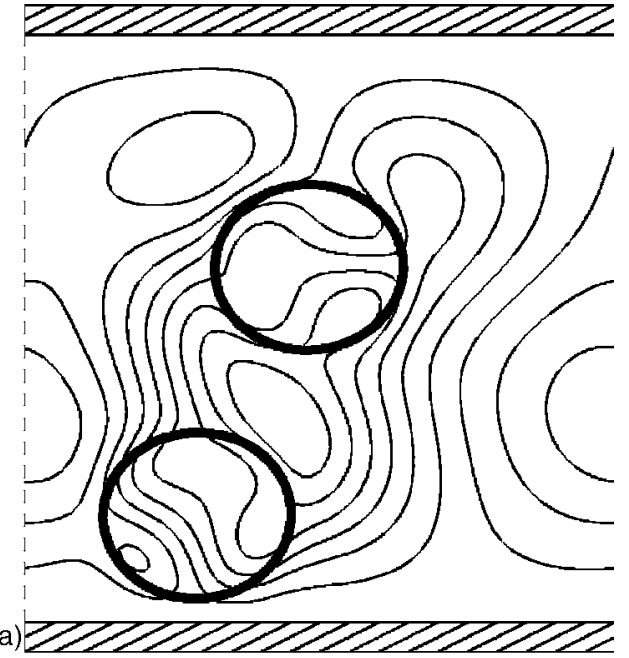

(a)
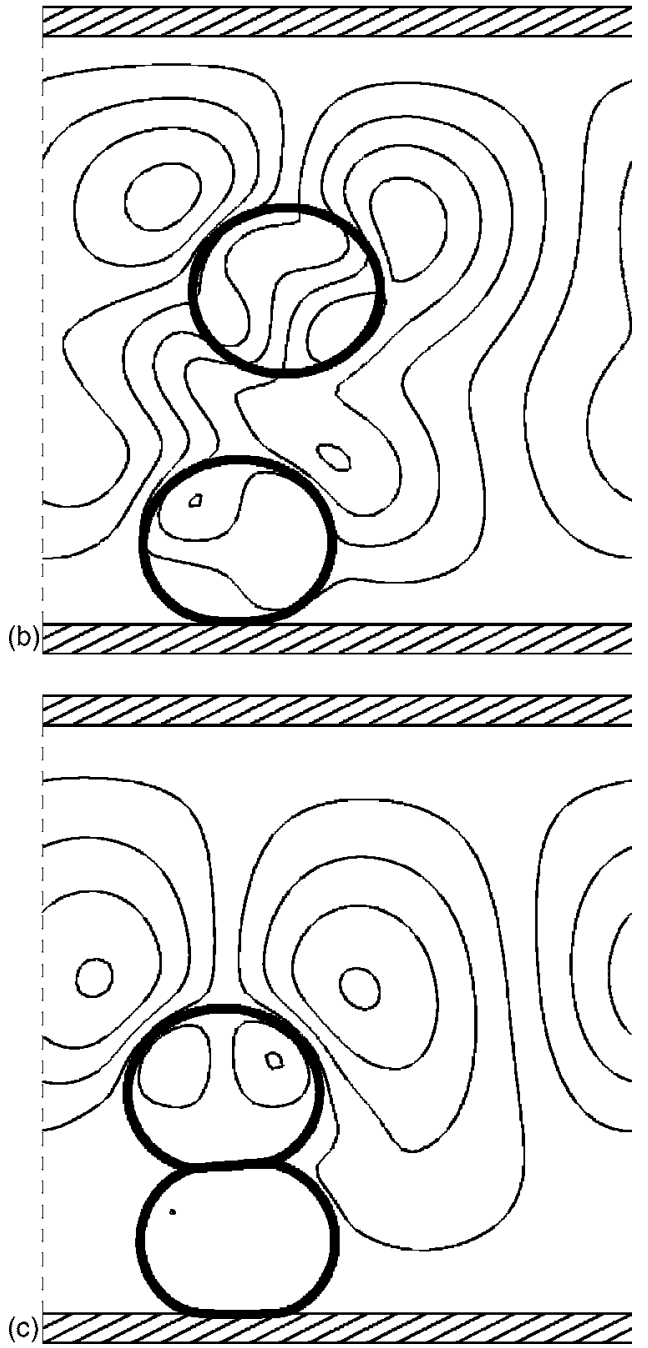

FIG. 3. The drops and streamlines for the interaction between two oblate drops. $S^{-1}=8.0, R=2.0$, and $C_{\mathrm{e}}=0.8$. Initial distance between the centroids of the drops is $r_{0}=3.0 a$.

oblate deformation, as well as using a higher $E$. These cases are shown in Fig. 1 using open circles. The evolution of the vertical and horizontal distances, for one particular initial condition, is plotted in Fig. 4(b) using discontinuous lines, along with the corresponding results from Fig. 4(a) (solid
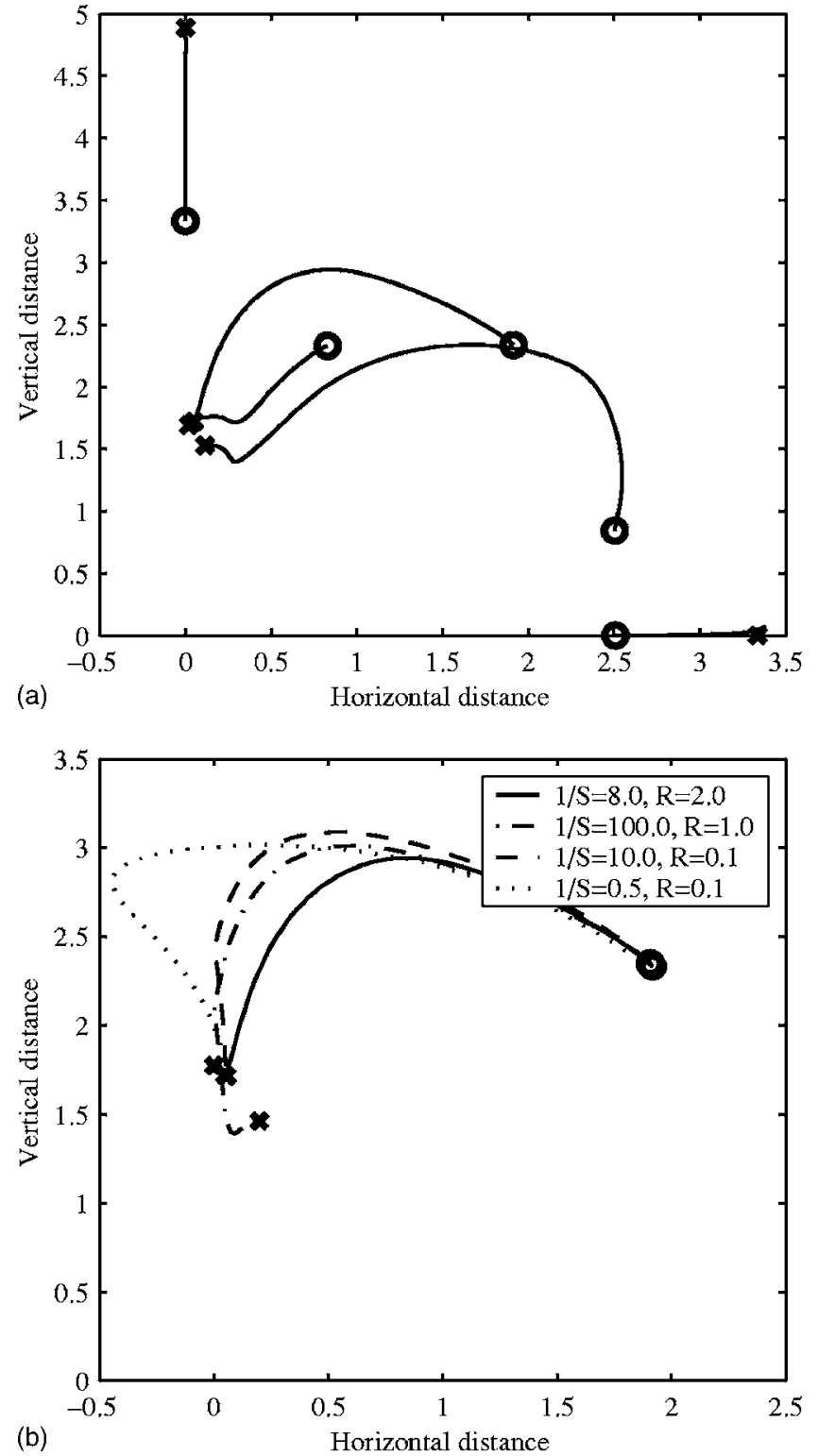

FIG. 4. (a) The vertical separation of the drops vs the horizontal separation for two-drop interactions. $S^{-1}=8.0, R=2.0$, and $C_{e}=0.8$. (b) The vertical separation of the drops versus the horizontal separation for two-drop interactions for different combinations of electrical properties.

line). In all cases the results show essentially the same behavior, once the time scale is rendered nondimensional. We note that for the oblate drops studied here, the dielectrophoretic attraction at close separations and the attraction induced by the fluid motion both collaborate to line the drops up along the electric-field lines. For prolate drops, on the other hand, the viscous fluid motion induces repulsion and the competition between the fluid-induced repulsion and the dielectrophoretic attraction can lead to more complex interactions.

\section{B. Drops in channel flow}

After examining the flow around a single drop and the interaction between two drops, we turn to the flow of an emulsion of drops in a channel. The distribution of drops in the channel is known to have a major impact on the flow 


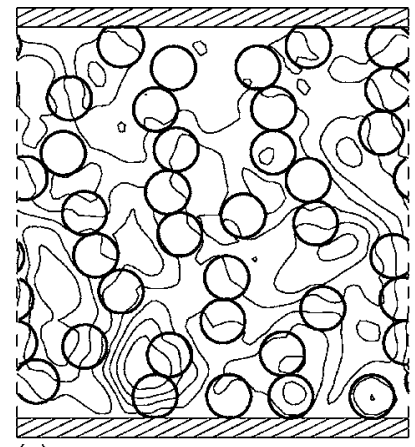

(a)

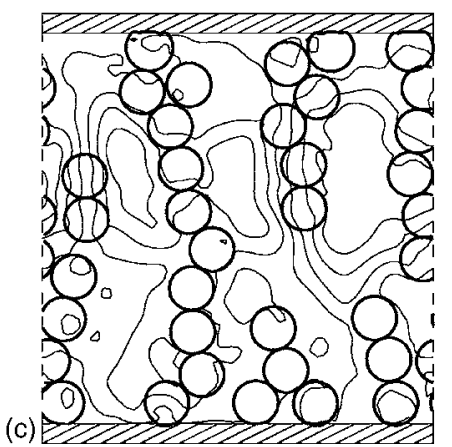

FIG. 5. The drops and the streamlines for the interaction of 36 drops in a Poiseuille flow at four times. $S^{-1}=8.0, R=2.0 ; 1 / E^{*}=0.0, C_{e}=0.06$, Oh $=0.2$, and $\alpha=0.36$. The flow in the channel is from the left to right.

rate. Here, we examine how the drop distribution changes as the pressure gradient is increased and the inverse of the nondimensional electrostatic force $\left(1 / E^{*}\right)$ is varied from 0 to 25 . This allows us to examine how the relative magnitude of the electrostatic forces and the fluid shear affects the drop configuration. In these simulations the density and viscosity ratios are 1.0, and the void fraction is 0.36 . This is a relatively high value and has been chosen in order to enhance the dropto-drop interaction. Later, this parameter is changed to study its influence. We start our analysis by using drops with the same electrical properties as in Sec. III A, where the conductivity ratio is 2.0 , and the permittivity ratio is 8.0. Initially, the applied pressure gradient is set to 0 and so that no external flow is imposed. The electric capillary number, however, is decreased to $C_{e}=0.06$ from Sec. III A, and later it is increased to study the influence of the deformability of the drops. The Ohnesorge (Oh) number is set to 0.2 and kept constant throughout all the simulations. Thirty-six drops are placed in a channel in a regular array that is perturbed by moving the drops slightly. No droplet coalescence is allowed here so the number of drops and their size remains constant throughout the simulation. Periodic conditions are imposed in the horizontal direction, so the drops that disappear through the right boundary reappear through the left one. On the top and the bottom walls, no-slip boundary conditions are applied and an electrical potential difference is imposed between the walls.

The drops and the streamlines are shown at three different times in Fig. 5 for the no-flow case. As seen in the twodrop case the drops attract each other in the direction parallel to the electric field. In the first frame $\left(t^{*}=57.0\right)$, some of the

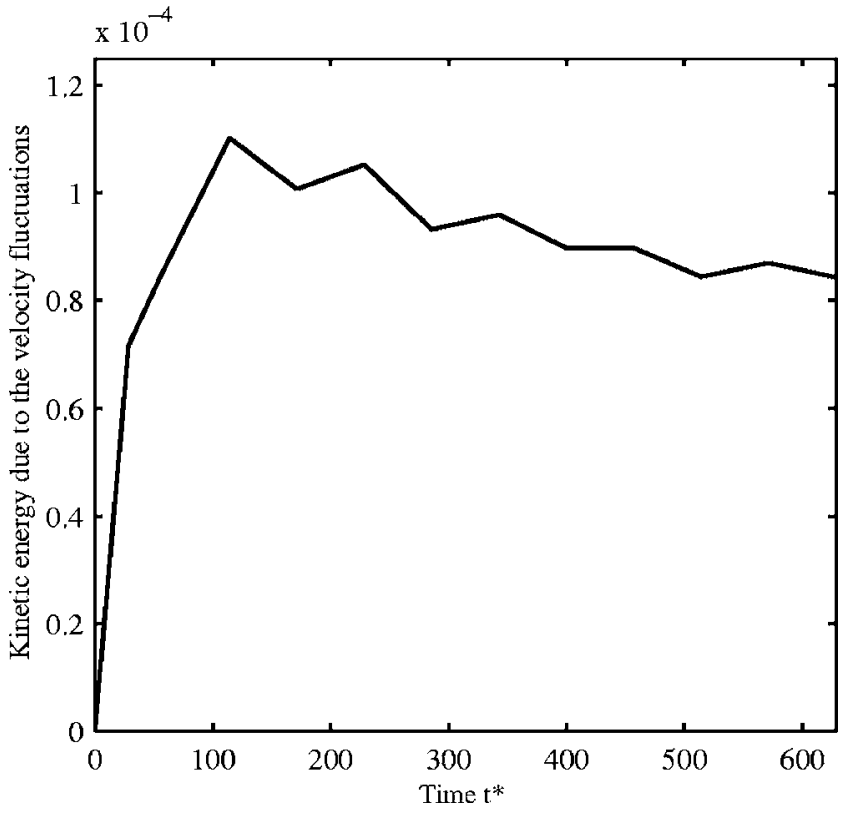

FIG. 6. The fluid kinetic energy for the simulation shown in Fig. 5.

drops close to the walls are moving to the walls. The drops in the middle of the channel attract each other and have started to form pairs or short fibers aligned with the electric field. The motion of the drops generates some fluid motion, consisting of weak eddies with a diameter of two or three drops. In the second frame, the droplet pairs have lined up into longer fibers and some of the fibers have been attached to droplets on the walls. This evolution continues in the third frame. In the final frame, all the drops are now part of a fiber consisting of several drops and essentially all the fibers are anchored at one wall or the other. One fiber spans the full distance between the top and the bottom wall. The flow field remains weak, but the size of the eddies has grown slightly. If the drops interacted only through dielectrophoric effects, or if the suspension were composed of solid particles, we would expect the fluid motion to slow down as the drops have all arranged themselves into fibers. For oblate drops, Pan and McKinley ${ }^{29}$ and Kimura et al $^{30}$ observed the formation of fibers aligned with the direction of the electric field. Their photographs were taken before any coalescence process occurred and showed a very similar microstructure to the results of our simulations. In Fig. 6 we plot the kinetic energy of the fluid (of both the drops and the suspending fluid) versus time, and while the fluid motion slows down slightly after the initial rise, it is clearly not going to 0 since the induced electrohydrodynamic stresses at the drop surface keep the fluid in motion, even after the drops stop.

In Fig. 7, where the drops and the streamlines are again shown at three different times, the flow rate has been increased $\left(1 / E^{*}=1.0\right)$. In the first frame some of the drops have been attracted to the walls, while short fibers appear in the middle of the channel. The fibers are, however, tilted by the fluid shear. The streamlines show that the velocity is still close to the initial parabolic profile. In the second frame more drops have become stuck on the wall and most of the drops in the middle belong to tilted fibers. While some of the 


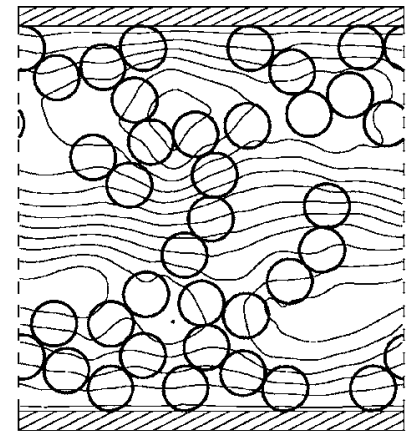

(a)

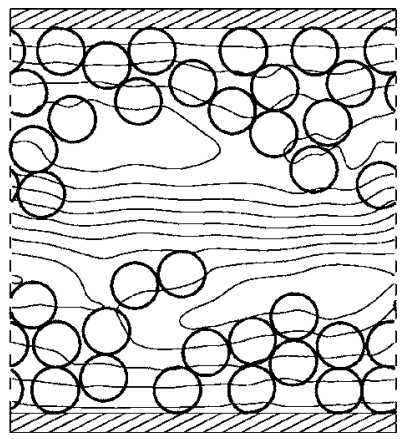

(b)

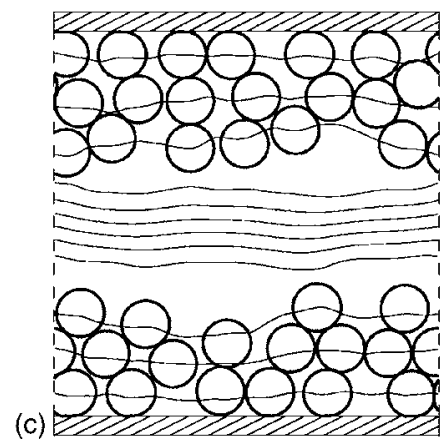

FIG. 7. The drops and the streamlines for the interaction of 36 drops in a Poiseuille flow at four times. $S^{-1}=8.0, R=2.0 ; 1 / E^{*}=1.0, C_{e}=0.06$, Oh $=0.2$, and $\alpha=0.36$. The flow in the channel is from the left to right.

fibers in the middle are connected to stationary drops on the walls, it is clear that these fibers are being stretched by the flow. In the third frame the stretched fibers have broken up and about half of the drops are in fibers that are connected to one wall and about half is connected to the other wall. The center of the channel is essentially free of drops. This process continues and eventually the drops pile up close to the walls. The streamlines show that at the late stage the drops at the walls are essentially stationary and the flow is confined to the narrow channel between the layers of drops. Once all the drops have been deposited on the walls, no further evolution of the drop distribution occurs and, if coalescence were allowed, we would expect to see a fluid film on each wall.

Figure 8 shows the drop distribution at a late time for $1 / E^{*}=25$. At this time the flow has reached an approximate steady-state. The flow rate is high enough so that the electrodynamic interactions are much weaker than the fluid shear and short fibers are immediately broken up. Drops are also prevented from sticking to the wall. Kimura et al. ${ }^{30}$ applied a constant shear to their polymer emulsion. They showed that, for oblate drops, the application of a strong shear led to the elongation of the drops and the breakup of the fibers in a similar fashion to our simulations. When $1 / E^{*}=4$ the drops form a layer on the walls, as for the $1 / E^{*}=1$ case, but the deposition along the walls occurs at a much slower rate as it is shown shortly when we present a few quantitative measures of the evolution. As it was pointed out in Sec. II, if no electric field is present the Reynolds and capillary numbers are used to determine the flow. In our cases, and taking the velocity at the center of the channel as our velocity scale, the

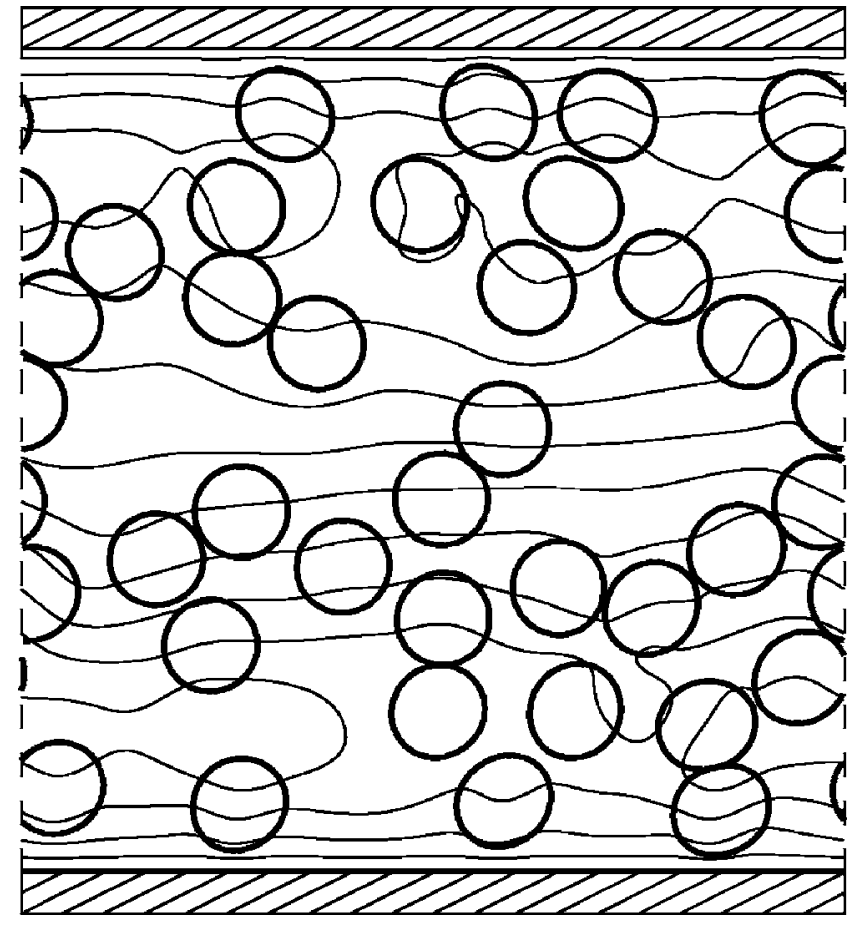

FIG. 8. The drops and the streamlines for the interaction of 36 drops in a Poiseuille flow at steady state. $S^{-1}=8.0, R=2.0 ; 1 / E^{*}=25.0, C_{e}=0.06, \mathrm{Oh}$ $=0.2$, and $\alpha=0.36$. The flow in the channel is from the left to right.

simulations shown here would result in a Reynolds number ranging from 0 to 100 . The capillary number, for a constant $\mathrm{Oh}=0.2$, ranges from 0 to 0.25 .

To quantify where the drops are located in the channel, the average nondimensional distance from the centerline is computed by

$$
|y|=\frac{1}{N_{d}} \sum_{i=1}^{N_{d}} \frac{y_{d}-(H / 2)}{H} .
$$

A value of $|y|$ close to a half indicates that the drops are mostly deposited on the walls while a low value shows that the drops are concentrated near the centerline. For drops in a uniform emulsion, obviously $|y|=0.25$. The temporal evolution of the nondimensional distance away from the centerline is shown for different nondimensional electrostatic forces in Fig. 9(a). When no initial pressure gradient is applied the drops form fibers that extend across the channel, but the average distance away from the centerline increases only slightly. As the mass flow is increased $\left(1 / E^{*}=1.0\right)$ the tendency of the drops to deposit along the walls leads to an increase in the nondimensional distance from the centerline. For this electrostatic force the maximum value obtained is 0.32 . If the drops coalesced and formed a flat layer near the walls, this distance would be 0.41 for the void fraction used here. The lowest value is achieved for $1 / E^{*}=25.0$ because no drop is deposited onto the walls and all the drops remain suspended.

The nondimensional distance away from the centerline is not sufficient to describe the drop distribution in the channel. For example, Fig. 9(a) shows that $|y|$ for the inverse of the nondimensional electrostatic forces of $1 / E^{*}=0$ and 

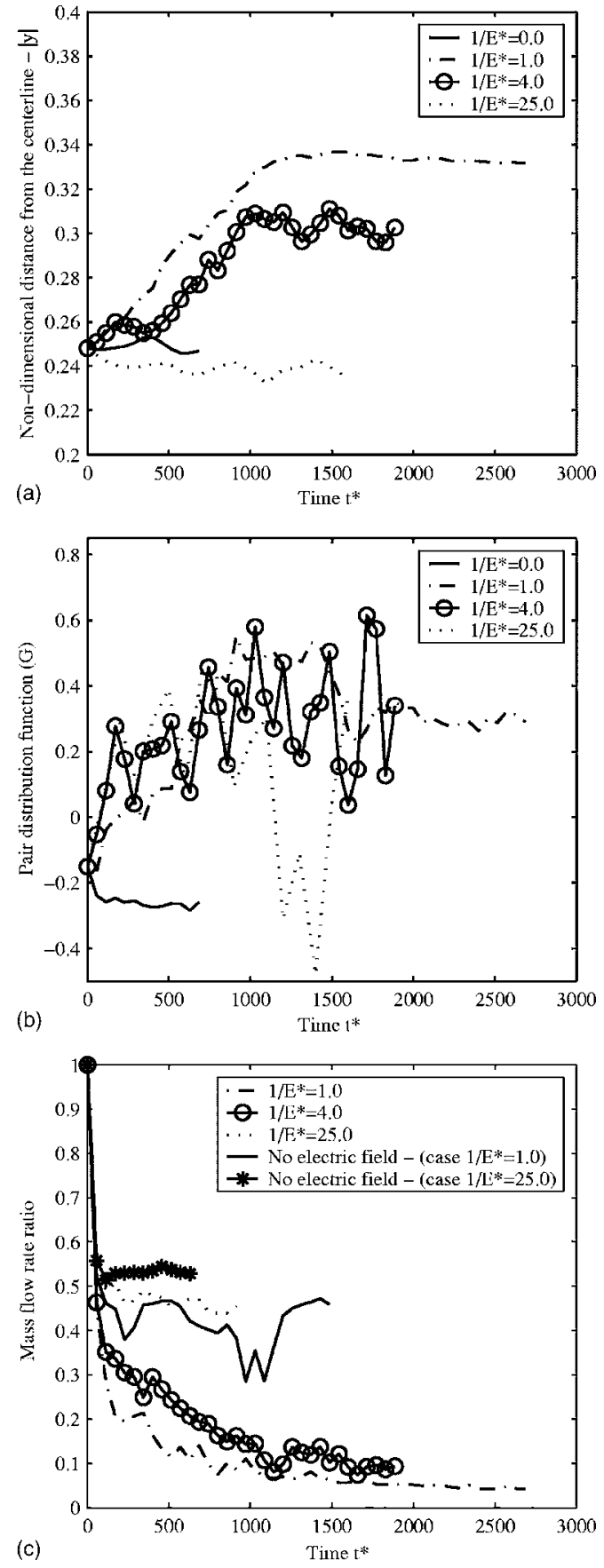

FIG. 9. (a) Nondimensional distance of the drops from the centerline $(|y|)$ vs time for different nondimensional electrostatic forces. $S^{-1}=8.0, R=2.0 ; C_{\mathrm{e}}$ $=0.06, \mathrm{Oh}=0.2$, and $\alpha=0.36$. (b) Relative position of the drops, as given by the pair distribution function $(G)$, vs time for different nondimensional electrostatic forces. $S^{-1}=8.0, R=2.0 ; C_{e}=0.06, \mathrm{Oh}=0.2$, and $\alpha=0.36$. (c) Mass flow rate ratio of the emulsion vs time for different nondimensional electrostatic forces. $S^{-1}=8.0, R=2.0 ; C_{e}=0.06, \mathrm{Oh}=0.2$, and $\alpha=0.36$.

$1 / E^{*}=25$ is essentially the same but the drop distribution in the channel is completely different. This makes it necessary to introduce another variable to describe the flow. For homogeneous flows the relative position of the drops has usually been quantified by the pair distribution function. While the pair distribution function is less suitable for nonuniform flows, it should be able to tell us something about how the drops are attracted to each other. In particular, when the drops form fibers or layers close to the walls, the pair distribution function is a good measurement of how the resulting structures look like. Here we find the weighted average of the distribution for drops that are close to each other. If $\theta_{i, j}$ is the angle between a line drawn connecting the centroids of drops $i$ and $j$ and the horizontal axis, we compute the weighted average by

$$
\mathrm{G}=\frac{1}{N_{\mathrm{cl}}} \sum_{i=1, N_{d}} \sum_{j=i+1, N_{d}} \cos \left(2 \theta_{i, j}\right) \quad \text { if } \quad\left|r_{i}-r_{j}\right|<3 a .
$$

Here, $N_{\mathrm{cl}}$ refers to the number of times two drops are separated by less than the critical distance, in this case three times the drop radius. If all the drops are stacked vertically, the value of $G$ is close to -1 and if the drops are lined up in horizontal rows the value is 1 . Note that here we average by $\cos \left(2 \theta_{i, j}\right)$ since the flow is two dimensional. For fully threedimensional flows the second Legendre polynomial is usually used to account for the difference in integrated volume near the poles and the equator of each drop. The evolution of the relative position of the drops, plotted in Fig. 9(b), shows that, when a pressure gradient is applied, $G$ increases due to the formation of layers close to the walls. However if no pressure gradient is applied, the formation of fibers leads to a decrease of $G$ until a value of about -0.3 is reached. At this moment the fibers span the distance between the walls and the flow is occluded.

The rheological properties of the fluid mixture is, obviously, strongly affected by the microscopic behavior of the drops. In Fig. 9(c) we plot the mass flow rate ratios versus time, for different nondimensional electrostatic forces. The mass flow ratio, or the inverse of the effective viscosity, is computed as the mass flow with and without drops for the same pressure gradient.

$$
\text { MFR }=\frac{Q_{\text {WITH DROPS }}}{Q_{\text {WITHOUT DROPS }}} .
$$

We note that we prefer to work with the mass flow ratio rather than the effective viscosity, since in two dimensions the fibration of the drops can block the flow completely, resulting in $M F R=0$. The effective viscosity, on the other hand, becomes infinitely large. In all cases the mass flow rate first decreases sharply due to the presence of the drops. For the intermediate case where the drops accumulate near the walls, the flow rate seems to reach an approximately steadystate value of slightly more than 0.2 , although long-time oscillations still may persist. If all the drops coalesced into a stationary layer at the wall, the flow rate in the narrower channel (for the same pressure gradient) would be reduced by a factor of 0.64 . Here the drops do not coalesce and the wall layer is both thicker and rougher, accounting for the larger reduction in flow rate. For the highest flow rate $\left(1 / E^{*}=25.0\right)$ the drops remain suspended and the flow rate is reduced by about half. We have repeated this simulation with the electric field turned off and find that the presence of drops leads to a very comparable reduction in flow rate $(0.54$ for no electric field versus 0.505 with the field). It seems, therefore, safe to conclude that for the electric-field strength used here the fluid shear is now the dominant effect, and that 

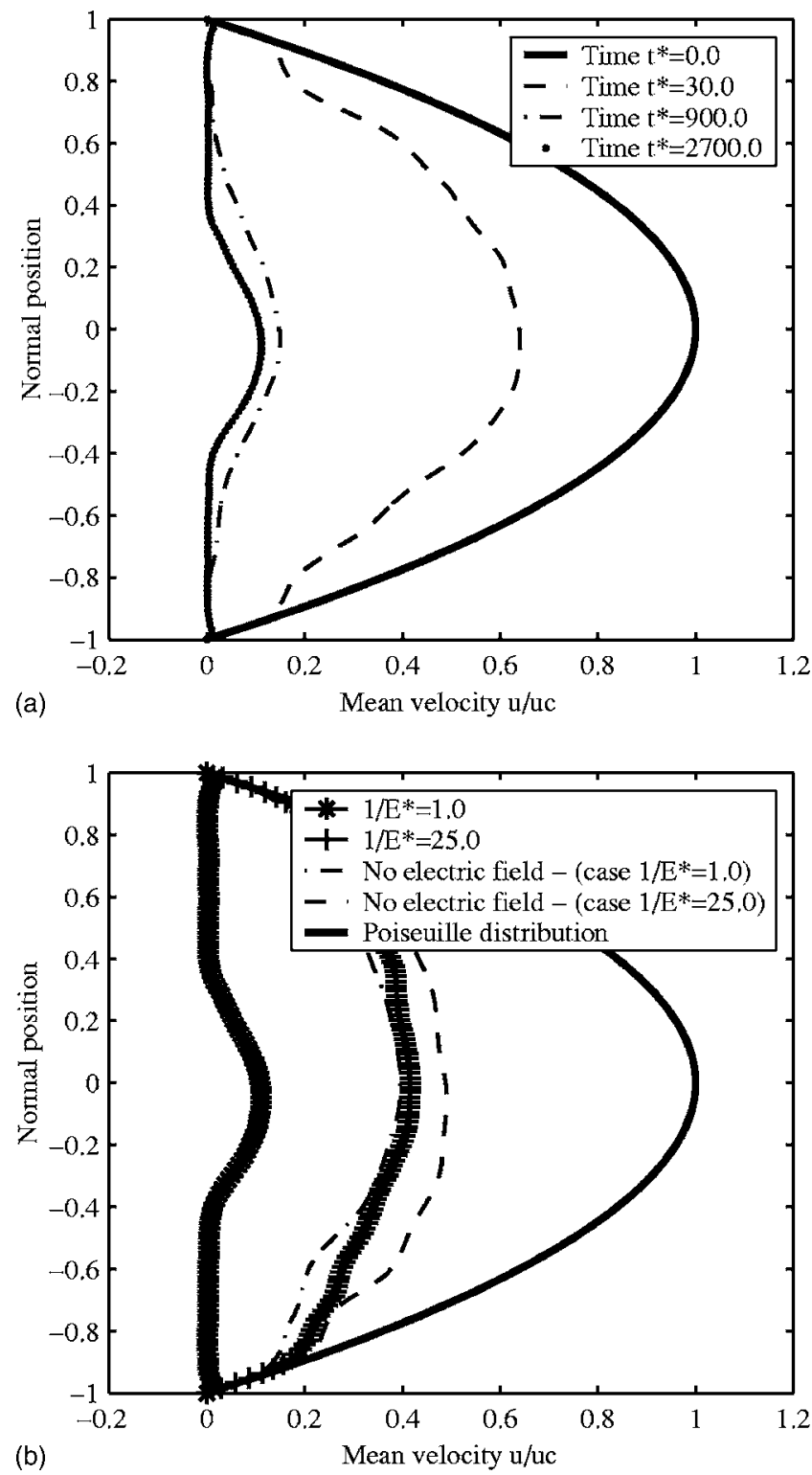

FIG. 10. (a) Evolution of the emulsion velocity profile. $S^{-1}=8.0, R=2.0$; $1 / E^{*}=1.0, C_{e}=0.06, \mathrm{Oh}=0.2$, and $\alpha=0.36$. (b) Comparison of the emulsion velocity profile for the cases with and without electric field at two different nondimensional electrostatic forces $-1 / E^{*}=1,25 . S^{-1}=8.0, R=2.0 ; C_{e}$ $=0.06$, $\mathrm{Oh}=0.2$, and $\alpha=0.36$.

the behavior of emulsions at higher flow rates would not be affected by the electric field.

To understand better the evolution of the flow, we show the temporal evolution of the mean velocity profile for $1 / E^{*}=1.0$ in Fig. 10(a). The evolution of the drop distribution with time for this case is shown in Fig. 7. When the drops are introduced and the electric field is turned on, the initial Poiseuille velocity profile is modified due to the presence of the drops. Initially, this modulation is stronger at the center of the channel. As the flow evolves, however, the deposition of the drops on the walls leads to a near-zero velocity in near the walls, but a parabolic velocity profile in the center of the channel. If no electric field was present, the drops would change the flow and reduce the flow rate, but in a different way and by not as much. The steady-state mean velocity profiles for the cases shown in Figs. 8 and 9 are compared to similar cases with no electric field in Fig. 10(b). When electrostatic forces dominate the interactions, the drops accumulate near the wall and a smaller amount of fluid flows between the drop layers, with a parabolic velocity profile. When the shear is stronger than the electrostatic interaction, $1 / E^{*}=25.0$, the drops remain suspended and lead to a flattening of the velocity profile, especially in the center of the channel where the drops accumulate. The drop interactions induced lead to a small reduction in the total flow rate, but overall the velocity profile is similar to the no electricfield case. Close to the walls there is almost no change since the wall shear has to balance the pressure gradient.

In Fig. 11 the velocity fluctuations, computed as the difference between the instantaneous velocity and the mean velocity found by averaging over planes normal to the wall, are plotted at three different times for the $1 / E^{*}=1.0$ case. The streamwise fluctuations are plotted in Fig. 11(a) and the normal velocity fluctuations are plotted in Fig. 11(b). Both the streamwise and the normal velocity fluctuations are reduced significantly as the flow evolves; the drops accumulate on the walls and the fluid passage becomes narrower. The locations of the fluctuation peaks move to where the shear is maximum $(r / H \approx \pm 0.4)$. In Fig. 11(c) we compare the streamwise fluctuating velocities for two different nondimensional electrostatic forces, $1 / E^{*}=1.0$ and $1 / E^{*}=25.0$, respectively, and the corresponding flow with the electric field turned off. The nondimensional fluctuations are of similar magnitude, although the positions where the maximum values appear change when the electric field is on. When the electric field is strong enough, the peaks move from close to the walls to the center of the channel.

For the systems examined here, the surface tension is sufficiently high (or the drops small enough and the electric field weak enough) so the drops remain nearly spherical. In this case, the evolution should be essentially independent of the electric capillary number and the Ohnsorge number. An increase in the electric-field strength makes the drop-to-drop and the drop-to-wall interactions stronger, and consequently speeds up the process. The speedup of the electric drop interactions is accounted for in the scaling of the time, but the ratio of the hydrodynamic and the electric time scale changes. For strong enough fields, we also expect the drops to deform. To examine how the strength of the electric field affects the flow we have simulated three different cases where the electric capillary number was increased from $C_{e}=0.06$ to $C_{e}=0.15,1.2$, and 3.0 , while the electric-field strength remained constant $\left(1 / E^{*}=1.0\right)$. In Figs. 12(a) and 12(b) we show the nondimensional distance from the centerline and the mass flow rate versus time. The curves in Fig. 12(a) for this value of the electric-field strength show that when the drops are nearly spherical $\left(C_{e}=0.15\right)$, the evolution is essentially similar (in nondimensional time) to the $C_{e}=0.06$ case. When the deformation is much higher, the nondimensional distance from the centerline is, in particular, higher since the deformation allows the drops to pack tighter at the wall, as shown in Fig. 13 for $C_{e}=3.0$. The mass flow rate ratio, see Fig. 12(b), shows a similar behavior. For spherical drops the flow rate decreases until the drops are 


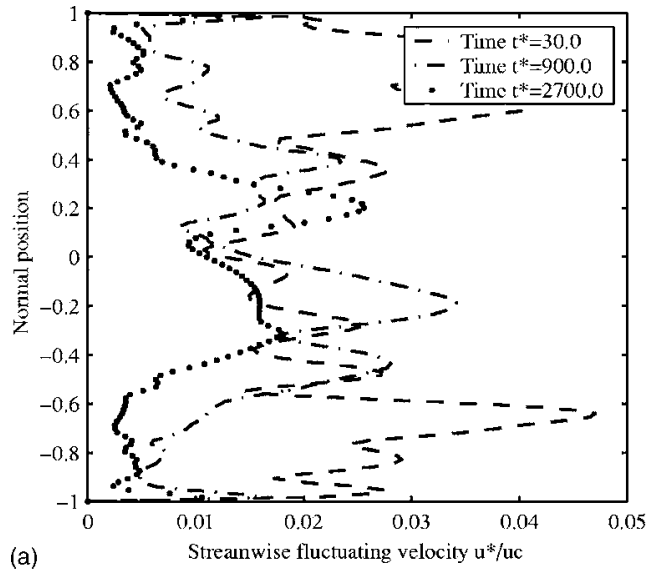

(a)
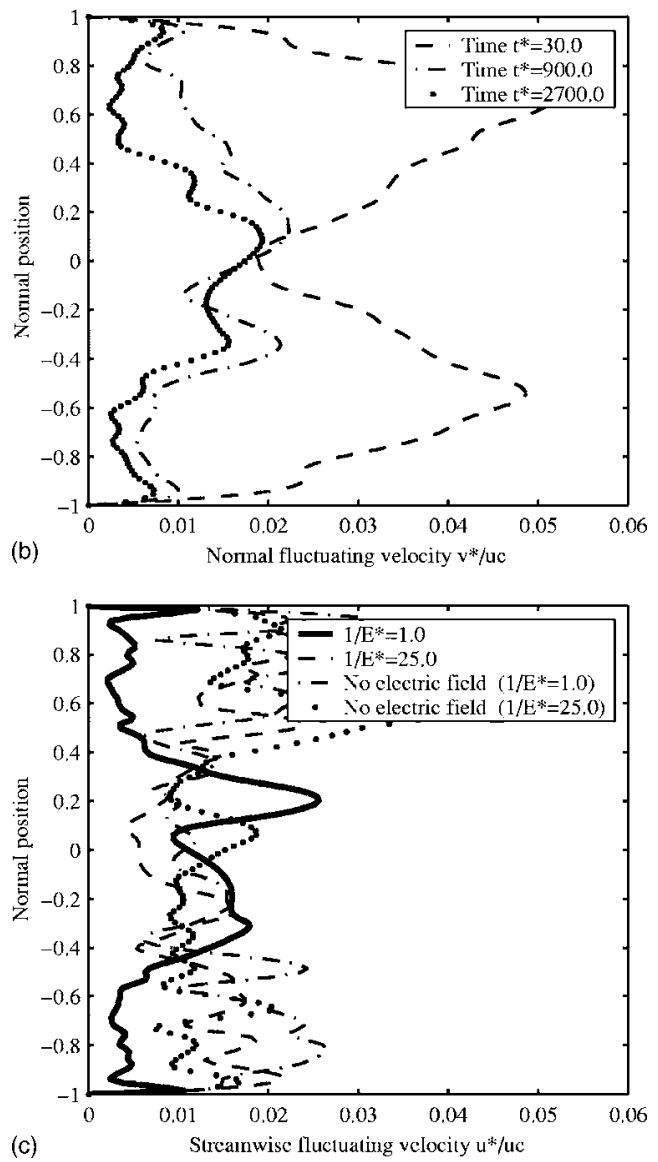

FIG. 11. (a) Evolution of the fluctuating streamwise velocity profile. $S^{-1}$ $=8.0, R=2.0 ; 1 / E^{*}=1.0, C_{e}=0.06, \mathrm{Oh}=0.2$, and $\alpha=0.36$. (b) Evolution of the fluctuating normal velocity profile. $S^{-1}=8.0, R=2.0 ; 1 / E^{*}=1.0, C_{e}$ $=0.06, \mathrm{Oh}=0.2$, and $\alpha=0.36$. (c) Comparison of the fluctuating streamwise velocity profile for the cases with and without electric field at two different nondimensional electrostatic forces $-1 / E^{*}=1,25 . S^{-1}=8.0, R=2.0 ; C_{e}$ $=0.06, \mathrm{Oh}=0.2$, and $\alpha=0.36$.

deposited on the walls. Then, it reaches an asymptotic value of approximately $5 \%$ of the original flow rate. For highly deformable drops $\left(C_{e}=3.0\right)$ the compact distribution of the drops on the walls leads to a lower friction, and therefore a higher mass flow rate, approximately $20 \%$ of the singlephase flow.

In the simulations presented so far the void fraction has been kept constant at 0.36 . The number of drops in the chan-
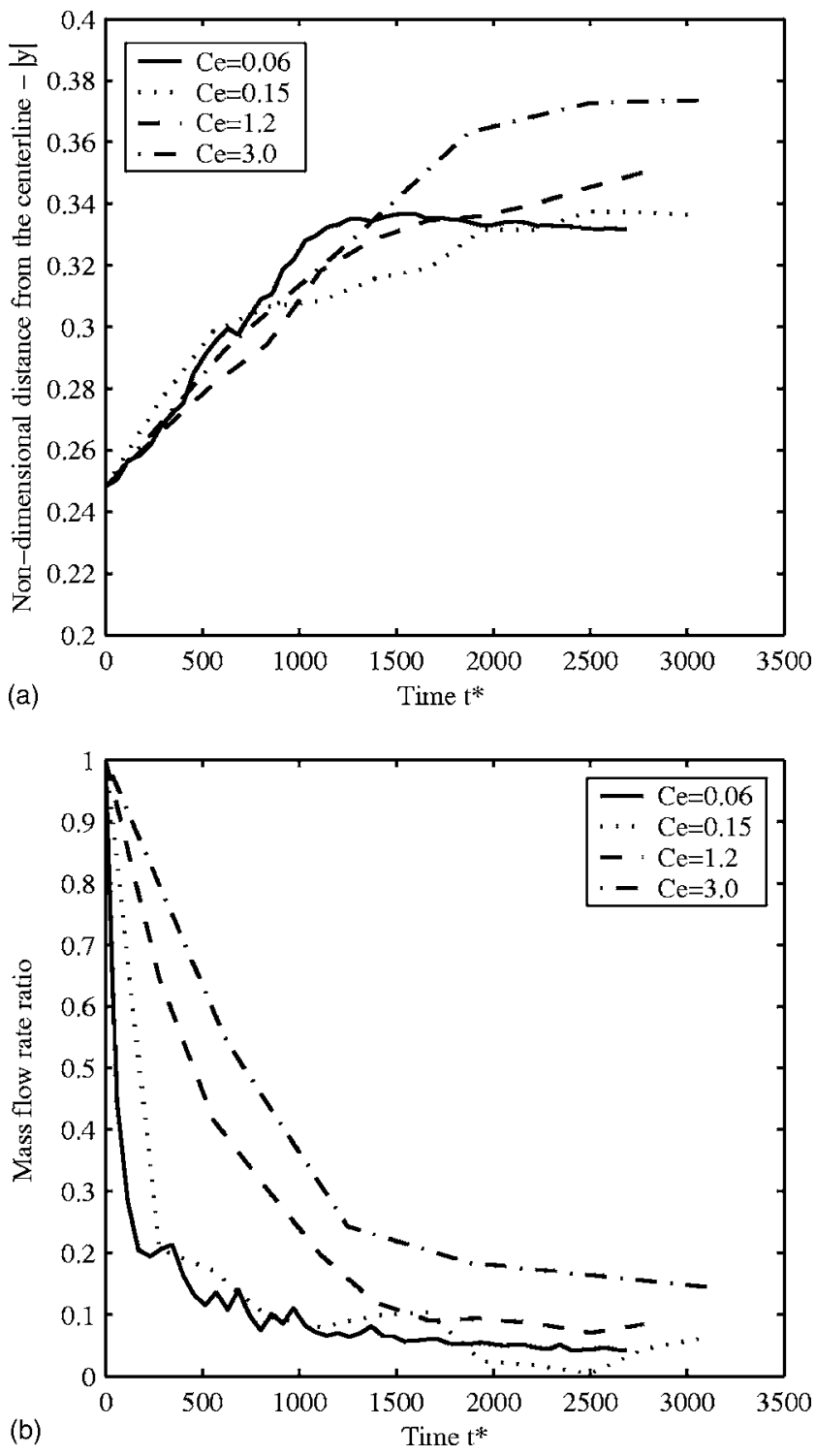

FIG. 12. (a) Nondimensional distance from the centerline $(|y|)$ vs time for different electric capillary numbers. $S^{-1}=8.0, R=2.0 ; 1 / E^{*}=1.0, \mathrm{Oh}=0.2$, and $\alpha=0.36$. (b) Mass flow rate ratio of the emulsion vs time. Evolution for different electric capillary numbers. Properties: $S^{-1}=8.0, R=2.0 ; 1 / E^{*}$ $=1.0, \mathrm{Oh}=0.2$, and $\alpha=0.36$.

nel and their size determine the void fraction. Here we keep the number of drops constant while varying the radius. Reducing the size of the drops decreases the probability of drop-to-drop or drop-to-wall interactions, so the electrohydrodynamic effects become weaker. For very low void fractions the electrostatic forces move some of the drops to the wall where they remain attached. However, the drops at the center of the channel are far away and do not "see" the drops on the walls. The deposition of drops onto the walls, therefore, slows down and some of the fibers remain suspended for a long time. Figures 14(a) and 14(b) show the drop distributions and streamlines at late time for void fractions of 0.24 and 0.44 , respectively. The nondimensional distance away from the centerline is shown in Fig. 15(a) and the mass flow rate in Fig. 15(b). For the higher void fraction, in addition to the deposition of drops on the walls, the emulsion 


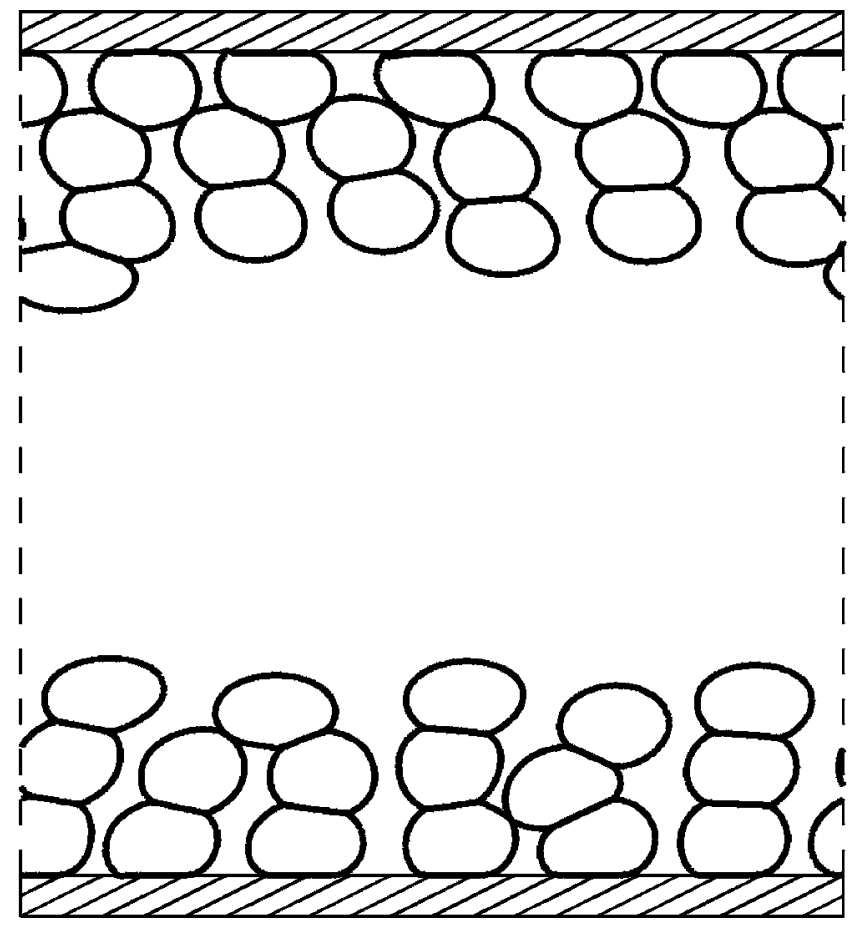

FIG. 13. Steady-state drop distribution for an emulsion of 36 highly deformable drops. $S^{-1}=8.0, R=2.0 ; 1 / E^{*}=1.0, C_{e}=3.0, \mathrm{Oh}=0.2$, and $\alpha=0.36$.

forms a cluster-like structure that spans the channel. The result is that the flow is almost blocked, and the mass flow rate, as shown in Fig. 15(b), reaches values very close to 0 , and during some instance even negative values. Visualization of the evolution of the drops showed that at some moments, the interaction between fibers was strong enough to push the flow backward, leading to a negative value of the mass flow rate ratio. This effect was found not only for oblate drops, but also in emulsions of prolate drops. For the lowest void fraction, $\alpha=0.24$, the drops eventually form layers on the walls. Because of the narrow layers, the resulting mass flow rate is approximately two times larger than the case with the medium void fraction $(\alpha=0.36)$.

In the simulations presented so far we have assumed that no coalescence takes place. In reality, drops would coalesce eventually. However, the experimental pictures of Pan and
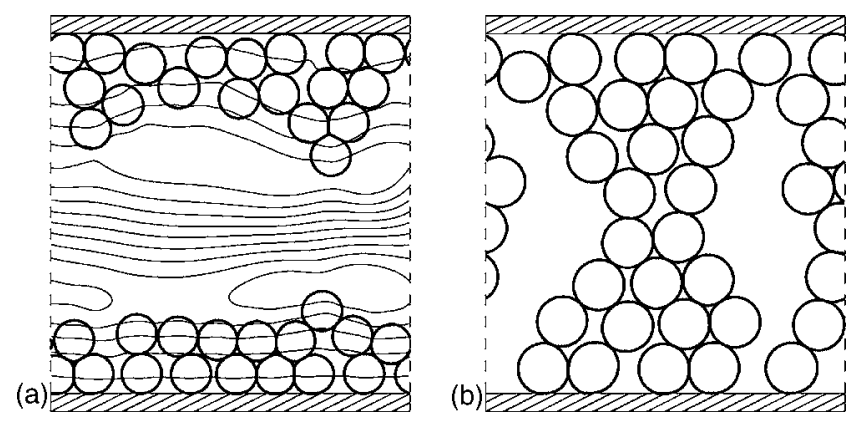

FIG. 14. (a) Steady-state drop distribution and streamlines for an emulsion of 36 drops at low void fraction. $S^{-1}=8.0, R=2.0 ; 1 / E^{*}=1.0, C_{e}=0.06$, $\mathrm{Oh}=0.2$, and $\alpha=0.24$. (b) Steady-state drop distribution and streamlines for a suspension of 36 drops at high void fraction. $S^{-1}=8.0, R=2.0 ; 1 / E^{*}=1.0$, $C_{e}=0.06$, $\mathrm{Oh}=0.2$, and $\alpha=0.44$.
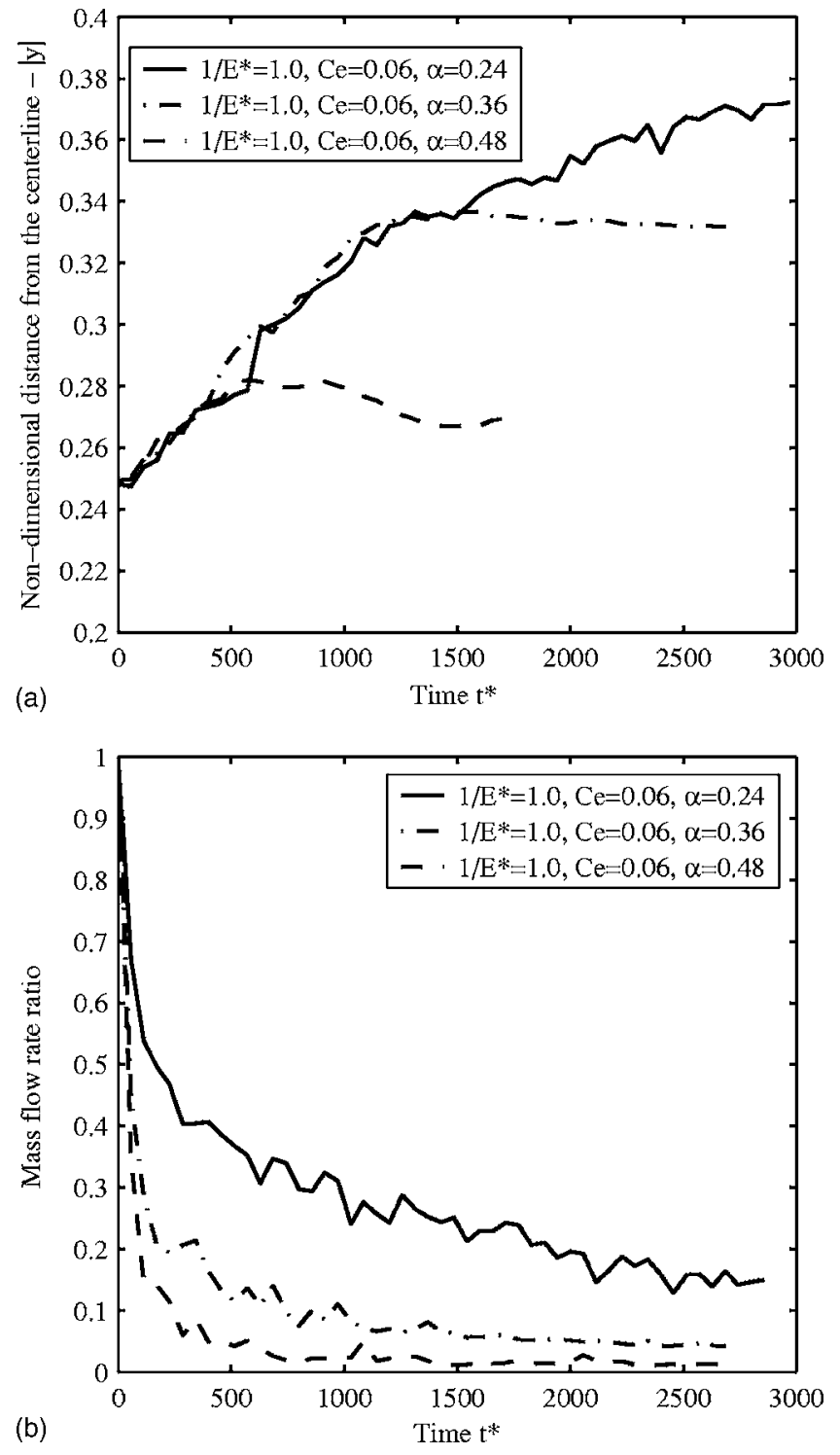

FIG. 15. (a) Nondimensional distance from the centerline $(|y|)$ vs time different void fractions. Properties: $S^{-1}=8.0, R=2.0 ; 1 / E^{*}=1.0, C_{e}=0.06$, and $\mathrm{Oh}=0.2$. (b) Mass flow rate ratio of the emulsion vs time for different void fractions. Properties: $S^{-1}=8.0, R=2.0 ; 1 / E^{*}=1.0, C_{e}=0.06$, and $\mathrm{Oh}=0.2$.

McKinley ${ }^{10}$ clearly show that coalescence is not instantaneous and droplet chains are clearly present. Thus, our steady-state results are only a quasisteady one, representing the state before coalescence.

\section{CONCLUSION}

An electric field applied to an emulsion of drops flowing through a channel can result in a significant modification of the distribution of drops across the cross section of the channel. Here, we have examined the effect of the flow rate for a relatively limited range of governing parameters, where the electric field induces a flow at the drop surface from the poles to the equator, and the drops become slightly oblate. In this parameter range, the induced viscous flow leads to an attraction between two drops whose centers lie on a line parallel to the electric field and a repulsion between two drops whose centers lie on a line perpendicular to the electric 
field. The flow, therefore, enhances the shorter-range dielectrophoretic interactions between drops. The results show that for low flow rate the drops form fibers that span the channel and block the flow. At higher flow rates short fibers form but the flow breaks up longer fibers and the drops accumulate at the walls, forming an essentially stationary layer. At even higher flow rates the flow shear prevents the formation of fibers and drops remain in suspension.

Although we have focused here on one particular value of the permittivity and conductivity ratio, simulations of other values in the oblate-drop regime suggest that the behavior described here is generic for all oblate drops. We have also elected to focus on nondimensional field strengths sufficiently weak so that drops remain nearly spherical and electroconvective effects remain negligible. In the limit of spherical drops (weak fields, small drops, or high surface tension), the number of nondimensional parameters is reduced by one and the field strength can be absorbed into a properly scaled time. We have shown that this scaling remains accurate even for fairly significant drop deformations. At higher-field values the drops break up and the dynamics can change significantly.

The investigation reported here has relied exclusively on examinations of two-dimensional systems. The interaction of the drops is, naturally, stronger than in fully threedimensional flows. Depending on whether the forces due to the electric stresses are stronger than the forces due to the pressure drop applied in the channel, or the pressure drop is stronger than the electric forces, three-dimensional emulsions still would exhibit the formation of fibers or a deposition of drops on the walls, respectively. However, an important difference between two- and three-dimensional situations is the rheological response of emulsions when the fibers appear. Whereas in two-dimensional flows the formation of fibers leads to an occlusion of the flow and to a zero flow rate, the formation of fibers in three-dimensional situations would not block the flow, as the suspending fluid could flow around the fibers. Thus, the presence of the electric field would substantially increase the effective viscosity of the emulsion, but a small flow rate would still exist.

The results suggested here might have practical implications for the transport of drop emulsions. The accumulation of drops at the channel walls, for example, suggests a way to separate drops from the suspending liquid. The results of the computations have been presented in nondimensional form, but the system examined in Fig. 6 could, for example, be realized by an emulsion of oil drops with a diameter of $0.5625 \mathrm{~mm}$ suspended in a silicon oil flowing through a channel of height $10 \mathrm{~mm}$ with a maximum centerline velocity of $23.36 \mathrm{~mm} / \mathrm{s}$ when there are no drops. The potential difference between the walls would have to be $900 \mathrm{~V}$.

Since the systems examined are two dimensional and the focus here has been on the qualitative effect of the flow on the drop distribution, we have not made much effort to examine in detail the structure of the drop distribution (beyond using a few simple measures). We conclude by noting that while all emulsions of oblate drops appear to show qualitatively the same behavior, prolate drops-where the dielectrophoretic forces and the flow-induced forces can oppose each other-show a much richer behavior, even for the same flow rate.

\section{ACKNOWLEDGMENTS}

This work was supported by the National Aeronautics and Space Administration through Grant Nos. NAG3-2332 and NAG3-2545.

${ }^{1}$ C. T. O'Konski and H. C. Thacher, Jr., "The distortion of aerosol droplets by an electric field," J. Phys. Chem. 57, 955 (1953).

${ }^{2}$ G. I. Taylor, "Disintegration of water drops in an electric field," Proc. R. Soc. London, Ser. A 280, 383 (1964).

${ }^{3}$ O. A. Basaran and L. E. Scriven, "Axisymmetric shapes and stability of isolated charged drops in an external electric field," Phys. Fluids A 1, 799 (1989).

${ }^{4}$ R. S. Allan and S. G. Mason, "Particle behaviour in shear and electric fields I. Deformation and burst of fluid drops," Proc. R. Soc. London, Ser. A 267, 45 (1962).

${ }^{5}$ G. I. Taylor, "Studies in electrohydrodynamics: I. The circulation produced in a drop by an electric field," Proc. R. Soc. London, Ser. A 291, 159 (1966).

${ }^{6}$ S. Torza, R. G. Cox, and S. G. Mason, "Electrohydrodynamic deformation and burst of liquid drops," Philos. Trans. R. Soc. London, Ser. A 269, 295 (1971).

${ }^{7}$ O. O. Ajayi, "A note on Taylor's electrohydrodynamic theory," Proc. R. Soc. London, Ser. A 364, 499 (1978).

${ }^{8}$ O. Vizika and D. A. Saville, "The electrohydrodynamic deformation of drops suspended in liquids in steady and oscillatory electric fields," J. Fluid Mech. 239, 1 (1992).

${ }^{9}$ F. Feuillebois, in Multiphase Science and Technology, edited by G. F. Hewitt, J. M. Delhaye, and N. Zuber (Hemisphere, New York, 1989), Vol. 4, p. 583

${ }^{10}$ J. Feng, H. H. Hu, and D. D. Joseph, "Direct simulation of initial value problems for the motion of solid bodies in a Newtonian fluid. II. Couette and Poiseuille flows," J. Fluid Mech. 277, 271 (1995).

${ }^{11}$ S. Mortazavi and G. Tryggvason, "A numerical study of the motion of drops in Poiseuille flow. Part 1. Lateral migration of one drop," J. Fluid Mech. 400, 1 (2000).

${ }^{12}$ B. P. Ho and L. G. Leal, "Inertial migration of rigid spheres in twodimensional unidirectional flows," J. Fluid Mech. 65, 365 (1976).

${ }^{13}$ P. Vasseur and R. G. Cox, "The lateral migration of a spherical particle in two-dimensional shear flows," J. Fluid Mech. 78, 385 (1976).

${ }^{14}$ R. G. Cox and S. K. Hsu, "The lateral migration of a solid particles in a laminar flow near a plane," Int. J. Multiphase Flow 3, 201 (1977)

${ }^{15}$ J. F. Brady and G. Bossis, "Stokesian dynamics," Annu. Rev. Fluid Mech. 20, 111 (1988).

${ }^{16}$ R. Nott and J. F. Brady, "Pressure-driven flow of suspensions: Simulation and theory," J. Fluid Mech. 275, 157 (1994).

${ }^{17} \mathrm{H}$. Zhou and C. Pozrikidis, "The flow of ordered and random suspensions of two-dimensional drops in a channel," J. Fluid Mech. 255, 103 (1993).

${ }^{18}$ M. Loewenberg and E. J. Hinch, "Numerical simulation of a concentrated emulsion in shear flow," J. Fluid Mech. 321, 395 (1996).

${ }^{19} \mathrm{X}$. Li and C. Pozrikidis, "Wall-bounded shear flow and channel flow of suspensions of liquid drops," Int. J. Multiphase Flow 26, 1247 (2000).

${ }^{20}$ D. L. Koch and R. J. Hill, "Inertial effects in suspension and porous media flows," Annu. Rev. Fluid Mech. 33, 619 (2001).

${ }^{21}$ W. M. Winslow, "Induced fibrillation of suspensions," J. Appl. Phys. 20, 1137 (1949)

${ }^{22}$ H. Block and J. P. Kelly, "Electro-rheology," J. Phys. D 21, 1661 (1988).

${ }^{23}$ T. C. Halsey, J. E. Martin, and D. Adolf, "Rheology of electrorheological fluids," Phys. Rev. Lett. 68, 1519 (1992)

${ }^{24}$ T. C. Halsey, "Electrorheological fluids," Science 258, 761 (1992).

${ }^{25}$ D. J. Klingerberg, F. van Swoi, and C. F. Zukoski, "Dynamic simulation of electrorheological suspensions," J. Chem. Phys. 91, 7888 (1989).

${ }^{26}$ D. J. Klingerberg, F. van Swoi, and C. F. Zukoski, "The small shear rate response of electrorheological suspension, I. Simulation if the point-dipole limit," J. Chem. Phys. 94, 6160 (1991).

${ }^{27}$ R. T. Bonnecaze and J. F. Brady, "Dynamic simulation of an electrorheological fluid,” J. Chem. Phys. 96, 2183 (1992). 
${ }^{28}$ P. A. Arp, R. T. Foister, and S. G. Mason, "Some electrohydrodynamic effects in fluid dispersions," Adv. Colloid Interface Sci. 12, 295 (1980).

${ }^{29}$ X. D. Pan and G. H. McKinley, "Characteristics of electrorheological responses in an emulsion system," J. Colloid Interface Sci. 195, 101 (1997).

${ }^{30}$ H. Kimura, K. Aikawa, Y. Masabuchi, J. Takimoto, K. Koyama, and T. Uemura, "Positive' and 'negative' electro-rheological effect of liquid blends," J. Non-Newtonian Fluid Mech. 76, 199 (1998).

${ }^{31}$ J. W. Ha and S. M. Yang, "Rheological responses of oil-in-oil emulsions in an electric field," J. Rheol. 44, 235 (2000).

${ }^{32}$ K. Tajiri, K. Ohta, T. Nagaya, H. Orihara, and A. Inoue, "Electrorheological effect in immiscible polymer blends," J. Rheol. 41, 331, (1997).

${ }^{33}$ K. Tajiri, H. Orihara, Y. Ishibashi, M. Doi, and A. Inoue, "Transient response of electrorheological effect to a step field in an immiscible polymer blend: First mode in type I blend," J. Rheol. 42, 335 (1998).

${ }^{34}$ K. Tajiri, H. Orihara, Y. Ishibashi, M. Doi, and A. Inoue, "Transient response of electrorheological effect to a step field in an immiscible polymer blend: First mode in type I blend," J. Rheol. 42, 335 (1998).

${ }^{35}$ H. Orihara, Y. Hosoi, K. Tajiri, Y. Ishibashi, M. Doi, and A. Inoue, "Electrorheological properties of a type-I immiscible polymer blend: Scaling and structural changes," J. Rheol. 43, 125 (1999).

${ }^{36}$ J. D. Sherwood, "Breakup of fluid droplets in electric and magnetic fields," J. Fluid Mech. 188, 133 (1988).

${ }^{37}$ T. Tsukada, T. Katayama, Y. Ito, and M. Hozawa, "Theoretical and experimental studies of circulation's inside and outside a deformed drop under a uniform electric field," J. Chem. Eng. Jpn. 26, 698 (1993).
${ }^{38}$ T. Tsukada, Y. Yamamoto, T. Katayama, and M. Hozawa, "Effect of an electric field on the behavior of a drop moving in a quiescent liquid," J. Chem. Eng. Jpn. 27, 662 (1994).

${ }^{39}$ J. Q. Feng and T. C. Scott, "A computational analysis of electrohydrodynamics of a leaky dielectric drop in an electric field," J. Fluid Mech. 311, 289 (1996)

${ }^{40}$ C. Sozou, "Electrohydrodynamics of a pair of liquid drops," J. Fluid Mech. 67, 339 (1975)

${ }^{41}$ J. C. Baygents, N. J. Rivette, and H. A. Stone, "Electrohydrodynamic deformation and interaction of drop pairs," J. Fluid Mech. 368, 359 (1998).

${ }^{42}$ J. R. Melcher and G. I. Taylor, "Electrohydrodynamics: A review of the role of interfacial shear stresses," Annu. Rev. Fluid Mech. 1, 111 (1969).

${ }^{43}$ D. A. Saville, "Electrohydrodynamics: The Taylor-Melcher leaky dielectric model," Annu. Rev. Fluid Mech. 29, 27 (1997).

${ }^{44}$ S. O. Unverdi and G. Tryggvason, "A Front tracking method for viscous incompressible flows." J. Comput. Phys. 100, 25 (1992).

${ }^{45}$ G. Tryggvason, B. Bunner, A. Esmaeeli, D. Juric, N. Al-Rawahi, W. Tauber, J. Han, S. Nas, and Y.-J. Jan, "A front tracking method for the computations of multiphase flow," J. Comput. Phys. 169, 708 (2001).

${ }^{46}$ C. S. Peskin, "Numerical analysis of blood flow in the heart," J. Comput. Phys. 25, 220 (1977).

${ }^{47}$ S. Krause and P. Chandratreya, "Electrorotation of deformable fluid droplets," J. Colloid Interface Sci. 206, 10 (1998).

${ }^{48}$ P. H. Rhodes, R. S. Snyder, and G. O. Roberts, "Electrohydrodynamic distortion of sample streams in continuous flow electrophoresis," J. Colloid Interface Sci. 129, 78 (1989).

${ }^{49}$ J. Che, Ph.D. dissertation, The University of Michigan (1999). 